\title{
Inclusion of Preferential Diffusion in Simulations of Premixed Combustion of Hydrogen/Methane Mixtures with Flamelet Generated Manifolds
}

\author{
Joost A. M. de Swart • Rob J. M. Bastiaans • \\ Jeroen A. van Oijen • L. Philip H. de Goey • \\ R. Stewart Cant
}

Received: 24 December 2009 / Accepted: 11 July 2010 / Published online: 27 July 2010

(C) The Author(s) 2010. This article is published with open access at Springerlink.com

\begin{abstract}
In this paper we study the possibility to account for preferential diffusion effects in lean turbulent premixed flames in numerical predictions with reduced chemistry. We studied the situation when hydrogen is added to methane at levels of $20 \%$ and $40 \%$ by volume in the fuel, at lean combustion $(\phi=0.7)$ with air. The base case of pure methane was used as a reference. In this case preferential diffusion effects are negligible. First the sensitivity of the mass burning rate to flame stretch was investigated, in one dimensional computations with detailed chemistry, to set reference values. Then the framework of the Flamelet Generated Manifolds (FGM) was used to construct an adequate chemical method to take preferential diffusion into account, without the need for using detailed chemistry. To that end a generalization of the method was presented in which five controlling variables are required. For this system, proper transport equations and effective Lewis numbers where derived. In practice not all five variables are necessary to include and as a first step we limited the amount in the numerical tests in this study to two controlling variables. The method was then tested in configurations in which there was an interaction of coherent vortices and turbulence with flames. It was demonstrated that a minimum of two controlling variables is needed to account for the changed mass burning rate as function of stretch and curvature. It was shown that one-dimensional FGM as well as one-step Arrhenius kinetics can not describe this relation.
\end{abstract}

J. A. M. de Swart · R. J. M. Bastiaans ( $₫)$ · J. A. van Oijen · L. P. H. de Goey

Combustion Technology, Department of Mechanical Engineering,

Eindhoven University of Technology, PO Box 513, 5600 MB Eindhoven, The Netherlands

e-mail:r.j.m.bastiaans@tue.nl

R. S. Cant

Engineering Department, Cambridge University, Trumpington Street,

Cambridge CB2 1PZ, UK

Present Address:

J. A. M. de Swart

TNO Science and Industry, PO Box 6235, 5600 HE Eindhoven, The Netherlands 
Keywords Turbulent combustion • Premixed flames • Preferent diffusion • Hydrogen

\section{Introduction}

With developments towards a sustainable energy supply and the reduction of greenhouse gases, the use of alternative fuels comes more and more into the picture. In this context there is progress towards the application of clean coal technology and the use of biomass. Both methods can result in the production of large amounts of hydrogen that can be used as a fuel. In many conventional power plants, electricity is generated by burning natural gas in a gas turbine.

Replacing a part of the natural gas by hydrogen influences the chemical processes significantly, because hydrogen is highly diffusive. This has an impact not only on the direct conversion rate of the fuel mixture but also on the stability of the flame which can be responsible for an increased integral conversion rate. The phenomenon associated with the fact that major species have different diffusion velocities, is referred to as preferential diffusion. Many experimental studies have been conducted to get insight in the associated phenomena, see e.g. [11, 14, 32].

Numerical simulation is a powerful tool. For fundamental investigations in which the interaction between small scale flow phenomena and the flame are important, all scales in the flow have to be resolved. Therefore, Direct Numerical Simulation (DNS) is the necessary tool to study preferential diffusion and its consequences. A drawback of DNS is that it is a computationally expensive method. In principle the chemical kinetics are taken into account by solving an equation for each species in the chemical system (detailed chemistry). This results in a very large and stiff system of equations and therefore the combination of DNS and detailed chemistry is hardly feasible. However DNS with detailed chemistry is not impossible. E.g. Hawkes and Chen [12] performed direct numerical simulations of premixed methane/hydrogen/air mixtures and used a reduced chemical model consisting of 19 chemical species and 15 reaction steps. Then, it is possible to use $N_{s}=19$ Lewis numbers, showing the relative diffusivities of all species, which results in a detailed and accurate solution. Sankaran et al. [23] performed simulations using detailed chemistry of premixed methane/air flames in a three-dimensional domain. Dunstan and Jenkins [5] used mixtures of hydrogen and methane in a two-dimensional premixed setting. Still, for each species a partial differential equation is solved and this is computationally very expensive.

In many DNS studies a single-step $R \rightarrow P$ (Arrhenius) chemical model is used $[1-3,13,22,28]$. A consequence of using single-step chemistry is that only a single effective Lewis-number is used. This single Lewis number is then defined as the ratio between diffusivity of heat and mass of a single species, usually the deficient reactant. This means that a local variation in enthalpy is captured, but local changes in element mass fractions can not be recovered. Local mass burning rates depend on the changes in enthalpy and elemental composition [10]. Therefore, it is expected that single-step chemistry is not able to predict the correct mass burning rate. Therefore additions that can account for enthalpy and element changes as a consequence of preferential diffusion will be studied in this paper. In real flames it is difficult to assign a single 
characteristic Lewis number because of the presence of many chemical species, each with a different Lewis number.

In this study, we investigate how the chemical reduction method FlameletGenerated Manifolds (FGM) can be used to capture the chemical kinetics. In the FGM-approach, also called Flamelet Prolongation of ILDM [8], as developed in $[17,19,29]$ the overall reaction progress is taken into account by solving an equation for one (or a few) reaction control variables. FGM reduces the number of equations to be solved and reduces the stiffness of the system of equations. The goal of the study presented in this paper is to include the effects of flame stretch and preferential diffusion on the burning velocity of premixed flames. To that end the method is generalized and implemented in the DNS-code SENGA, [15]. The generalization is made to include elemental composition and enthalpy. It is found that it is a good first step to use $2 \mathrm{D}$ manifolds, where the first dimension describes the overall reaction progress and the second dimension is used to capture changing local conditions, e.g. due to preferential diffusion.

We consider fuel mixtures consisting of methane and hydrogen where the molar hydrogen content in the fuel is equal to $X_{\mathrm{H}_{2}}=0.0, X_{\mathrm{H}_{2}}=0.2$ and $X_{\mathrm{H}_{2}}=0.4$. Combustion takes place under lean conditions $(\phi=0.7)$ with air as oxidizer under atmospheric conditions. Cases of vortex-flame interaction are studied as well as flames subjected to turbulent flows. Results are assessed by looking at local and global properties. Locally flamelets are extracted and stretch and curvature fields are used to evaluate burning speeds with increasing dimension of the chemistry. Also the flamelet hypothesis and numerical errors are evaluated. Globally the ensemble of burning speeds versus flame stretch is evaluated to determine Markstein numbers and integral burning rates and flame surface are studied. Detailed chemistry (which is easily feasible for one-dimensional cases) is used to support results and to show differences between the different simulation approaches.

In an earlier attempt by Vreman et al. [30] FGM was used to resolve the thermodiffusive instabilities. In that study only the different diffusion speeds of heat and fuel were taken into account. But differential diffusion between species also plays a role. Here we model the integral differential diffusion effects more rigorously. Essentially multi-dimensional manifolds are required to capture local deviations in the (elemental) composition and enthalpy. In the present study it is concluded that a 2D manifold can capture the major effects of preferential diffusion. Moreover it is shown that the combination of DNS and FGM, including preferential diffusion effects is computationally feasible and accurate. This is a first step towards statistical modeling with Large Eddy Simulations (LES) and Reynolds-Averaged NavierStokes (RANS) methods that would be required for practical design calculations.

The paper is organized as follows. To introduce some reference flame data, first stretched one-dimensional flames are considered. Here we use detailed chemistry to investigate the response of the flame to stretch. Subsequently a system of controlling variables is introduced that generalizes the FGM parametrization. Then the governing equations for these variables are addressed, defining effective Lewis numbers. This is followed by a description of manifold creation and selection of the final controlling variables, of which the number is deliberately limited to two. This is followed by the introduction of the physical problem of vortex-flame interaction. Results are shown, analyzed and discussed. We then extend the research to a turbulent problem after which we end with the conclusions. 


\section{Stretched One-Dimensional Flames with Detailed Chemistry}

In this section a first analysis is presented on the behaviour of one-dimensional premixed flames including preferential diffusion effects. First theory is presented, supplied by results of detailed calculations of lean methane-hydrogen mixtures. Both are based on the flamelet equations as presented in de Goey and ten Thije Boonkkamp [10]. Flamelet equations are the governing equations projected on a coordinate system attached to the flame. Therefore the one-dimensional coordinate is defined as the trajectory normal to the gradients in a premixed flame. In this section we will consider adiabatic one-dimensional flames with different fuels and stretch rates. For diffusion transport modeling the constant Lewis number approach is used. In this approach the diffusivity is taken to be constant and expressed in terms of the conductivity, but for each species it has a different value. This is consistent with our modeling work that we describe later in the paper.

Hydrogen addition will influence the sensitivity of the mass burning rate to flame stretch. Let us recall Eq. (49) of [10], where the stretched mass burning rate, $m=\rho s_{L}$, is written at the burnt side. This mass burning rate, originally defined at the burnt side, can be rewritten at the inner layer (subscript il) as

$$
\frac{m_{i l}}{m_{i l}^{0}}=1-\frac{K a_{\mathcal{Y}, i l}}{L e_{\mathcal{Y}}}+\Delta h_{i l} \frac{\partial}{\partial h_{i l}^{0}}\left(\ln m_{i l}^{0}\right)+\sum_{j=1}^{N_{e}-1} \Delta Z_{j, i l} \frac{\partial}{\partial Z_{j, i l}^{0}}\left(\ln m_{i l}^{0}\right),
$$

ignoring higher order terms. Here a superscript 0 indicates unstretched conditions, $h$ is the enthalpy and $Z_{j}$ is the element mass fraction of element $j$, for $N_{e}$ elements. A flame progress is introduced with $\mathcal{Y}$, being a combination of chemical species such that it is monotonically increasing. It is normalized, being 0 at the unburned side and 1 at burnt conditions. This solution is based on an analysis of the flamelet equations, Eqs. (20) of [10], with the Karlovitz integral defined as

$$
K a_{\mathcal{Y}, i l}=\frac{L e_{\mathcal{Y}}}{m_{i l}^{0}} \int_{s_{u}}^{s_{b}} \rho K\left[\mathcal{Y}-H\left(s-s_{i l}\right)\right] \mathrm{d} s,
$$

with $H\left(s-s_{i l}\right)$ being the Heaviside function equal to zero when the distance along the flame $s<s_{i l}$ and one otherwise. The mass based flame stretch rate is $K=\frac{1}{M} \frac{d M}{d t}$ for an element of mass $M$, moving with the flame, see [9]. The preferential diffusion effect, indicated by the two terms on the right hand side of (1) is related to changes in enthalpy, $\Delta h_{i l}$ and element mass fractions $\Delta Z_{j, i l}$ and depends linearly on flame stretch, being zero at zero stretch. The preferential diffusion terms can be written as linear functions of $K a_{\mathcal{Y}, \text { il }} / L e_{\mathcal{Y}}$,

$$
\Delta h_{i l} \frac{\partial}{\partial h_{i l}^{0}}\left(\ln m_{i l}^{0}\right)+\sum_{j=1}^{N_{e}-1} \Delta Z_{j, i l} \frac{\partial}{\partial Z_{j, i l}^{0}}\left(\ln m_{i l}^{0}\right)=C K a_{\mathcal{Y}, i l} / L e_{\mathcal{Y}},
$$

which yields

$$
\frac{m_{i l}}{m_{i l}^{0}}=1-(1-C) \frac{K a_{\mathcal{Y}, i l}}{L e_{\mathcal{Y}}}=1-\mathcal{M} \frac{K a_{\mathcal{Y}, i l}}{L e_{\mathcal{Y}}},
$$

and defines the Markstein number $\mathcal{M}=1-C$. The Markstein number describes the sensitivity of the mass burning rate to flame stretch. Since higher order terms are not 
Table 1 Properties of unstretched laminar flames with different fuel mixture, $\phi=0.7$

\begin{tabular}{llllll}
\hline & $X_{\mathrm{H}_{2}}$ & $X_{\mathrm{CH}_{4}}$ & $m^{0}\left[\mathrm{~kg} / \mathrm{m}^{2} \mathrm{~s}\right]$ & $T_{a d}[\mathrm{~K}]$ & $\delta_{f}^{0}[\mathrm{~m}]$ \\
\hline Mixture A & 0.0 & 1.0 & 0.218 & 1,844 & $6.77 \cdot 10^{-4}$ \\
Mixture B & 0.2 & 0.8 & 0.250 & 1,855 & $6.12 \cdot 10^{-4}$ \\
Mixture C & 0.4 & 0.6 & 0.300 & 1,871 & $5.37 \cdot 10^{-4}$ \\
\hline
\end{tabular}

taken into account when defining the Markstein number, the slope around $K=0$ should be taken as the Markstein number. However, since sometimes it is difficult to take the slope around $K=0$ and approximately linear profiles are observed, in this study the Markstein number is taken as the slope of a complete set of data-points that is considered. The Markstein number is equal to one, when there is no preferential diffusion $\left(L e_{i}=1\right)$.

Furthermore it must be recognized that a small value of the Karlovitz integral does not necessarily indicate small stretch rates. Even in the thin reaction zones regime stretch might heavily fluctuate through the preheat and reaction zone (see e.g. [18]). However, positive and negative values of the flame stretch easily cancel and result in a small Karlovitz integral while local flame stretch rates can be large. For weak stretch (and small variance through the flame), it is justified to neglect the higher order terms and the effect of preferential diffusion is captured by the Markstein number. However, when stretch rates become larger, higher order $\mathrm{Ka}$ terms may become important and it is not certain wether the relation as given in (4) yields unique Markstein numbers. This will be investigated in later sections of this paper.

To study the effects of preferential diffusion in one-dimensional flames a series of flames is simulated by using the one-dimensional code CHEM1D [4, 25], with detailed chemistry. Three different methane/hydrogen fuel mixtures are considered, where the mole fraction of hydrogen $X_{\mathrm{H}_{2}}$ is $0.0,0.2$ and 0.4 , respectively. The stoichiometry is taken to be $\phi=0.7$ and all flames are calculated with the GRI3.0 mechanism, [24]. The properties of these flames are shown in Table 1. It is shown that when the hydrogen content in the fuel increases, the mass burning rate and the adiabatic temperature increase while the flame thickness decreases. The increase in mass burning rate is caused by an increase in flame temperature and an increased supply of active radicals, as shown in e.g. [7, 32].

The density, the temperature and the reaction progress variable of the onedimensional unstretched laminar flames are compared in Fig. 1. For these variables,
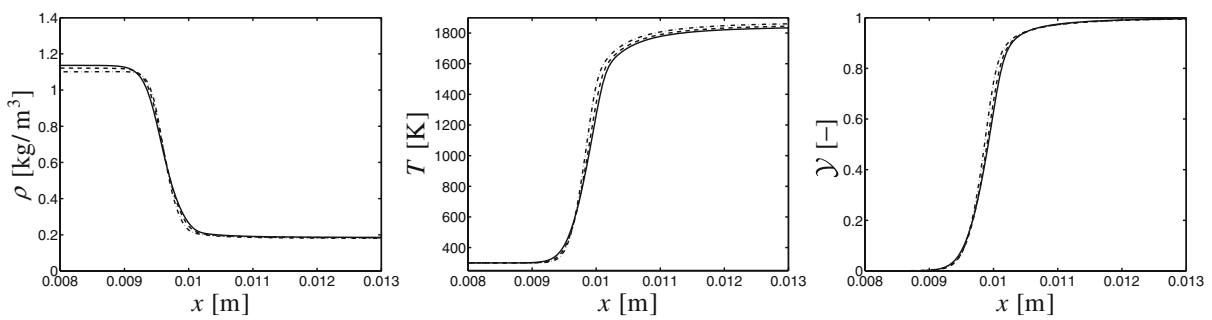

Fig. 1 Profiles of density, temperature and progress variable of one-dimensional laminar SENGAFGM simulations with full line: $X_{\mathrm{H}_{2}}=0.0$, dashed line: $X_{\mathrm{H}_{2}}=0.2$ and dash-dotted line: $X_{\mathrm{H}_{2}}=0.4$ 
Fig. 2 Inner layer mass burning rate as a function of Karlovitz integral for different methane/hydrogen mixtures using detailed chemistry; full line: $X_{\mathrm{H}_{2}}=0.0$, dashed: $X_{\mathrm{H}_{2}}=0.2$, dash-dotted: $X_{\mathrm{H}_{2}}=0.4$

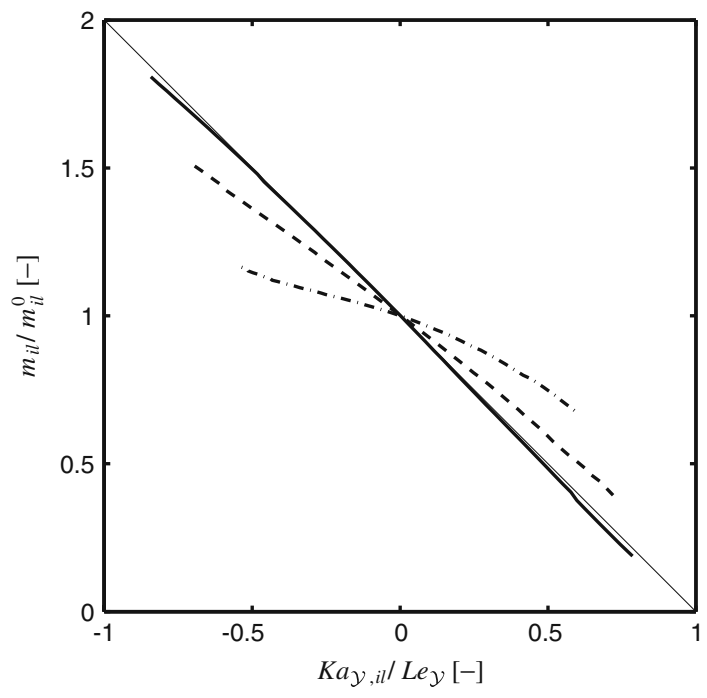

only small differences exist between the three fuel mixtures, although the mass burning rate changes significantly. Density and adiabatic temperature differ and the profiles of all three variables are steeper when more hydrogen is present in the fuel, resulting in a smaller flame thickness.

To find the Markstein numbers of the mixtures presented in Table 1, flame stretch and curvature are applied in such a way that the mass burning rate is constant through the flame. This is explained more thoroughly in Section 3.3 and (16). The Markstein number can then be found by plotting the scaled inner layer mass burning rate $m_{i l} / m_{i l}^{0}$ versus the scaled Karlovitz integral $K a_{\mathcal{y}}$,il $/ L e_{y}$. This is shown in Fig. 2. One can observe that the absolute slopes become smaller when $X_{\mathrm{H}_{2}}$ in the fuel increases. The global slopes for the three cases are $-1.01,-0.77$ and -0.40 , respectively. Taking local slopes at $K a y, i l / L e y=0$, yields $-1.02,-0.75$ and -0.35 , respectively. This shows that for weak stretch the slope around $K a_{\mathcal{Y}, i l} / L e_{y}=0$ in this flame stretch range is close to the slope of the whole data-set. It becomes clear that the pure methane case shows almost no preferential diffusion effect but an increasing hydrogen content in the fuel mixture shows a larger preferential diffusion effect.

Concluding, it was shown that preferential diffusion effects are large for hydrogen addition. This means that in a computational approach, preferential diffusion effects have to be accounted for. How this is done in the framework of FGM, is explained in the following section.

\section{FGM Implementation}

\subsection{A generic system of controlling variables}

When performing multi-dimensional DNS of turbulent combustion using detailed chemistry, multi-species transport is taken into account. This is an accurate way of incorporating the effects of flame stretch and preferential diffusion on the flame 
behavior. A DNS-simulation using detailed chemistry results in a very large and stiff system of equations. However, not all timescales are equally important. This is substantiated by a number of publications $[6,16,31]$, where time scale analysis was performed for different combustion systems looking at the reaction path in composition space. It was found that the reaction path in composition space is embedded in a low-dimensional manifold, which can be described by a small number of variables. Moreover, the chemical equilibrium state is described completely by the element composition, the total enthalpy and the pressure. When flame stretch and preferential diffusion are present, the elements and enthalpy are locally redistributed. Combustion then takes place under locally changing conditions (i.e. changing elemental composition and enthalpy). Therefore, in order to capture flame stretch and preferential diffusion, enthalpy, elemental composition and pressure have to be known.

In order to predict an accurate mass burning rate, we need one or more progress variables to describe the path to the chemical equilibrium state. In this work, only one reaction progress variable $\mathcal{Y}$ is used. We also need element mass fractions $Z_{j}$ and enthalpy $h$ to describe the chemical equilibrium. Consistently with this observation, instead of using detailed chemistry and solving partial differential equations for all species, in the FGM-framework five controlling variables should be used for a complete description. The first controlling variable is the reaction progress variable, $Y_{c v 1}=\mathcal{Y}$. The other controlling variables, $Y_{c v 2}$ to $Y_{c v 5}$, are used to capture the local elemental composition and enthalpy, $Z_{C}, Z_{O}, Z_{H}$ and $h$. Thus, the vector of controlling variables is given by

$$
\mathbf{Y}_{c v}=\left[\begin{array}{c}
\mathcal{Y} \\
Z_{C} \\
Z_{O} \\
Z_{H} \\
h
\end{array}\right] .
$$

Variables of interest are stored and looked up depending on 5 entries, i.e. $Y_{c v 1}$ to $Y_{c v 5}$. The element mass fraction of nitrogen $Z_{N}$ follows from the unity complement of elements, $\sum_{j=1}^{N_{e}} Z_{j}=1$.

The controlling vector as shown in (5) is the general case, where all elements can change independently. It is also possible to use combinations of element mass fraction and enthalpy as a controlling variable. This can be an efficient alternative because element mass fractions and enthalpy can be highly correlated. Therefore to construct an independent set of controlling variables with a decreasing sensitivity on the solution is a way to limit to control the controlling vector by taking only the first few most sensitive ones. In the present approach we will take only one additional variable first.

\subsection{Governing equations}

In the present study the equations that describe the density, energy and velocity are solved by the SENGA code, [15]. Apart from that, additional equations for the controlling variables are implemented and solved to describe the chemical composition. In this section, the conservation equations for species, reaction progress variable, other controlling variables and enthalpy are derived. At the end a definition 
of the Lewis number of any controlling variable will follow and in particular the Lewis number of the progress variable $L e_{\mathcal{y}}$ is given.

A transport equation for the reaction progress variable $\mathcal{Y}$ is derived from conservation of species $Y_{i}$. Conservation of species with Fickian diffusion is given by

$$
\frac{\partial\left(\rho Y_{i}\right)}{\partial t}+\nabla \cdot\left(\rho \mathbf{u} Y_{i}\right)=\nabla \cdot\left(\frac{\lambda}{L e_{i} c_{p}} \nabla Y_{i}\right)+\dot{\rho}_{i}
$$

The species diffusion contribution can be split in two parts, which show nonpreferential diffusion and preferential diffusion explicitly, as

$$
\frac{\partial\left(\rho Y_{i}\right)}{\partial t}+\nabla \cdot\left(\rho \mathbf{u} Y_{i}\right)-\nabla \cdot\left(\frac{\lambda}{c_{p}} \nabla Y_{i}\right)=\nabla \cdot\left(\frac{\lambda}{c_{p}}\left(\frac{1}{L e_{i}}-1\right) \nabla Y_{i}\right)+\dot{\rho}_{i} .
$$

The first term on the right hand side shows the preferential diffusion contribution, i.e. it only has a contribution when $L e_{i} \neq 1$.

A next step is to use the definition of the reaction progress variable,

$$
\mathcal{Y}=\sum_{i=1}^{N_{s}} \alpha_{i} Y_{i}
$$

where $\alpha_{i}$ are constants. This yields

$$
\frac{\partial(\rho \mathcal{Y})}{\partial t}+\nabla \cdot(\rho \mathbf{u} \mathcal{Y})-\nabla \cdot\left(\frac{\lambda}{c_{p}} \nabla \mathcal{Y}\right)=\nabla \cdot\left(\frac{\lambda}{c_{p}} \sum_{i}^{N_{s}} \alpha_{i}\left(\frac{1}{L e_{i}}-1\right) \nabla Y_{i}\right)+\dot{\rho} \mathcal{Y},
$$

where $\dot{\rho} \mathcal{Y}=\sum_{i}^{N_{s}} \alpha_{i} \dot{\rho}_{Y_{i}}$. Using that $Y_{i}=Y_{i}\left(\mathcal{Y}, Y_{c v 2}, \ldots, Y_{c v 5}\right)$ and applying the chain rule, the preferential diffusion term (first term on r.h.s.) can be written as

$$
\begin{aligned}
\nabla & \left(\frac{\lambda}{c_{p}} \sum_{i}^{N_{s}} \alpha_{i}\left(\frac{1}{L e_{i}}-1\right) \nabla Y_{i}\right) \\
& =\nabla \cdot\left(\frac{\lambda}{c_{p}} \sum_{i}^{N_{s}} \alpha_{i}\left(\frac{1}{L e_{i}}-1\right)\left(\frac{\partial Y_{i}}{\partial \mathcal{Y}} \nabla \mathcal{Y}+\frac{\partial Y_{i}}{\partial Y_{c v, j}} \nabla Y_{c v, j}\right)\right)
\end{aligned}
$$

where a repeated index $j$ implies summation for $j=2,5$. It can be seen that the preferential diffusion contribution depends on the gradients of $\mathcal{Y}$ and $Y_{c v, j}$. As a last step, it is assumed that locally the controlling variables $Y_{c v, j}$ are given by the manifold relation $Y_{c v, j}=Y_{c v, j}^{1 D}(\mathcal{Y})$, which is a function of $\mathcal{Y}$ only. Instead of using all species, we take the overall reaction progress into account by the first controlling variable $Y_{c v 1}=\mathcal{Y}$. This results in the following equation for conservation of the reaction progress variable,

$$
\frac{\partial(\rho \mathcal{Y})}{\partial t}+\nabla \cdot(\rho \mathbf{u} \mathcal{Y})=\nabla \cdot\left(\frac{\lambda}{c_{p}} \nabla \mathcal{Y}\right)+\nabla \cdot(d \mathcal{Y} \nabla \mathcal{Y})+\dot{\rho} \mathcal{Y} .
$$

with

$$
d \mathcal{Y}=\frac{\lambda}{c_{p}} \sum_{i=1}^{N_{s}}\left(\frac{1}{L e_{i}}-1\right) \alpha_{i}\left(\frac{\partial Y_{i}}{\partial \mathcal{Y}}+\frac{\partial Y_{i}}{\partial Y_{c v, j}} \frac{\partial Y_{c v, j}^{1 D}}{\partial \mathcal{Y}}\right)
$$


Consequently, generalizing the derivation, it turns out that conservation of the full set of controlling variables can be written as

$$
\frac{\partial\left(\rho \mathbf{Y}_{c v}\right)}{\partial t}+\nabla \cdot\left(\rho \mathbf{u} \mathbf{Y}_{c v}\right)=\nabla \cdot\left(\frac{\lambda}{c_{p}} \nabla \mathbf{Y}_{c v}\right)+\nabla \cdot\left(\mathbf{d}_{Y_{c v}} \nabla Y_{c v 1}\right)+\dot{\rho}_{Y_{c v}} .
$$

where $\mathbf{d}_{Y_{c v}}$ represents the preferential diffusion coefficient vector and $\dot{\rho}_{Y_{c v}}$ is the chemical source term vector. This source term has a value for the progress variable but is zero for the element mass fractions and enthalpy. The value of $\alpha_{i}$ in $\mathbf{d}_{Y_{c v}}$ is replaced by $w_{j i}$ for each element fraction $j$, and $h_{i}$ for the enthalpy equation. The preferential diffusion coefficient and source terms are stored in the flamelet database.

Now, the Lewis number of the progress variable can be defined by rewriting the preferential diffusion $\nabla \cdot\left(d_{\mathcal{Y}} \nabla \mathcal{Y}\right)$ term as,

$\nabla \cdot\left(\frac{\lambda}{c_{p}} \sum_{i=1}^{N_{s}} \alpha_{i}\left(\frac{1}{L e_{i}}-1\right)\left(\frac{\partial Y_{i}}{\partial \mathcal{Y}}+\frac{\partial Y_{i}}{\partial Y_{c v, j}} \frac{\partial Y_{c v, j}^{1 D}}{\partial \mathcal{Y}}\right) \nabla \mathcal{Y}\right)=\nabla \cdot\left(\frac{\lambda}{c_{p}}\left(\frac{1}{L e_{\mathcal{Y}}}-1\right) \nabla \mathcal{Y}\right)$,

and then the 'Lewis number' of the progress variable is defined,

$$
\frac{1}{L e_{\mathcal{Y}}}-1=\sum_{i=1}^{N_{s}} \alpha_{i}\left(\frac{1}{L e_{i}}-1\right)\left(\frac{\partial Y_{i}}{\partial \mathcal{Y}}+\frac{\partial Y_{i}}{\partial Y_{c v, j}} \frac{\partial Y_{c v, j}^{1 D}}{\partial \mathcal{Y}}\right)
$$

Therefore the Lewis number of the progress variable $L e_{\mathcal{y}}$ depends on the definition of the reaction progress variable $\mathcal{Y}$ and that it is not constant, although the individual species Lewis numbers are taken as constants. Note that for a progress variable consisting of a single species the Lewis number will attain the value of this species transport. How the Lewis number varies will be studied in Section 3.4, where the (current) reaction progress variable is defined.

\subsection{Manifold creation}

In order to create the manifold detailed flamelets are calculated and the resulting data that are relevant for continuing the simulation and/or postprocessing is stored in a table. The key entries in the manifold are the controlling variables. The selection of controlling variables is described in the next section. Here we discuss the conditions of the flamelets and the data that is stored. Note that the ensemble of conditions should match the realizations of phenomena that occur in the simulations. In principle this can be selected independently from the selection of controlling variables, besides the realizability constraints on $\mathcal{Y}_{c v}$.

For the present purposes the manifold is spanned by laminar stretched premixed one-dimensional flames, simulated using detailed chemistry with the numerical flame code CHEM1D [4, 25], at relevant conditions for later application. Since we want to incorporate the effect of flame curvature and flame stretch we have to take these degrees of freedom into account in the generation of the manifold. However there are multiple ways to do this; we can not decide a priori which combinations of stretch and curvature will occur. Furthermore we want to extend the manifold only with one single parameter. We choose a situation in which the mass burning rate along the 
flamelets is constant $(\partial m / \partial s=0)$ at a certain applied curvature $(\kappa=1 / \sigma \quad \partial \sigma / \partial s=$ Const.). Using mass conservation from the flamelet equations, [10], this yields for the flame stretch field

$$
\rho(s) K(s)=-m \kappa .
$$

From the flamelet simulations, the variables that are required in the DNS simulations are stored in a database. In the simulations presented in this paper, the chemical source term of the first controlling variable $\dot{\rho}_{Y_{c v 1}}$ and the preferential diffusion coefficients of the controlling variables $d_{Y_{c v}}$ are stored. Additionally the specific heat $c_{p}$, the mixture gas constant $R_{g}$ and the heat conductivity $\lambda$ are added to the manifold database to be used in the simulation as well. The term $h-c_{p} T$ is stored and used to compute the temperature from the equation for energy, which is defined as

$$
e=\sum_{\alpha=1}^{N} Y_{\alpha} h_{\alpha}-\frac{p}{\rho}+\frac{1}{2} u_{i} u_{i}
$$

In order to take acoustic effects into account, the temperature, $T$, can not be taken from the (incompressible) database. It is assumed that the enthalpy of the mixture can be written as $\sum_{\alpha=1}^{N} Y_{\alpha} h_{\alpha} \approx h_{f g m}+c_{p, f g m}\left(T-T_{f g m}\right)$, which is valid for small $T-T_{\text {fgm }}$. This means that the energy can be written as

$$
e=h_{f g m}-c_{p, f g m} T_{f g m}+c_{p, f g m} T-R_{g, f g m} T+\frac{1}{2} u_{i} u_{i},
$$

where it was used that $p / \rho=R_{g, f g m} T$, with $R_{g, f g m}$ the mixture gas constant. Now the term $h_{f g m}-c_{p, f g m} T_{f g m}$ is stored in the flamelet database and the temperature can be found by solving an algebraic equation,

$$
T=\frac{e-\left(h-c_{P} T\right)_{f g m}-\frac{1}{2} u_{k} u_{k}}{c_{p, f g m}-R_{g, f g m}},
$$

where the fgm-subscript indicates that these variables come from the flamelet database. Additionally, the mass fraction of methane is stored and used for flamelet analysis when post-processing the results.

In the next subsections proper controlling variables are selected. This is performed in such a way that the first controlling variable is associated with the main 1D structure of the flame, which can be considered as the principle flame coordinate. Therefore the first controlling variable is also referred to as the progress variable. As mentioned earlier, besides the progress variable, we will define only one additional controlling variable to include preferential diffusion effects to first order.

\subsection{The progress variable (first control variable)}

The first controlling variable, or the reaction progress variable, is chosen as

$$
\mathcal{Y}^{*}=\frac{Y_{\mathrm{CO}_{2}}}{M_{\mathrm{CO}_{2}}}+\frac{Y_{\mathrm{H}_{2} \mathrm{O}}}{M_{\mathrm{H}_{2} \mathrm{O}}}-X_{\mathrm{H}_{2}}^{u} \frac{Y_{\mathrm{H}_{2}}}{M_{\mathrm{H}_{2}}}-\left(1-X_{\mathrm{H}_{2}}^{u}\right) \frac{Y_{\mathrm{CH}_{4}}}{M_{\mathrm{CH}_{4}}}
$$

where $X_{\mathrm{H}_{2}}^{u}$ is the mole fraction of hydrogen in the (unburnt) fuel. All major species are represented in the reaction progress variable. The mass fractions of $\mathrm{CO}_{2}$ and $\mathrm{H}_{2} \mathrm{O}$ are included to ensure that $\mathcal{Y}$ is continuously increasing [17]. The mass fraction 

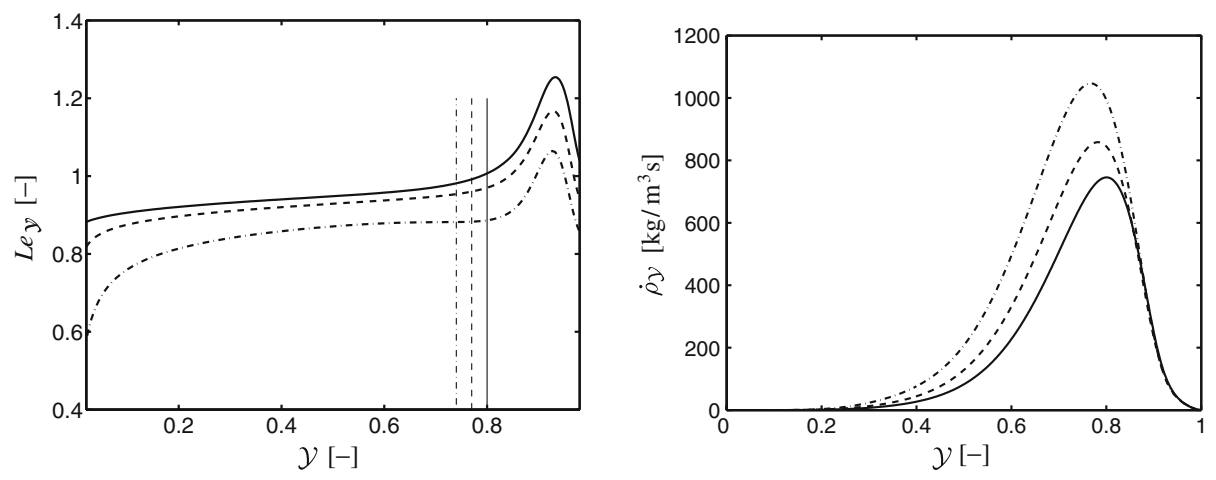

Fig. 3 Left: Lewis number of the progress variable as a function of progress variable with full line: $X_{\mathrm{H}_{2}}=0.0$, dashed line: $X_{\mathrm{H}_{2}}=0.2$ and dash-dotted line: $X_{\mathrm{H}_{2}}=0.4$. Vertical lines: indicate inner layer position for each case. The $L e y$ decrease from 1.01 to 0.96 and 0.88 for increasing amount of hydrogen. Right: Chemical source term as a function of the first controlling variable with full line: $X_{\mathrm{H}_{2}}=0.0$, dashed line: $X_{\mathrm{H}_{2}}=0.2$ and dash-dotted line: $X_{\mathrm{H}_{2}}=0.4$

of $\mathrm{CH}_{4}$ is included to capture the first stage of the chemical reactions in the flame. Hydrogen is only present in the reaction progress variable when it is present in the fuel. The progress variable is then scaled with the maximum and minimum value of the unstretched flamelet,

$$
Y_{c v 1}=\mathcal{Y}=\frac{\mathcal{Y}^{*}-\mathcal{Y}_{\text {min }}^{*}}{\mathcal{Y}_{\text {max }}^{*}-\mathcal{Y}_{\text {min }}^{*}} .
$$

The Lewis number of the progress variable $L e y$ generally depends on position, see (15). This is shown in Fig. 3 (left), where the Lewis number of the progress variable $\operatorname{Ley}_{\mathcal{Y}}(\mathcal{Y})$ is plotted. When no hydrogen is present the Lewis number at the inner layer position (the position of the maximum source term, indicated by vertical lines) is approximately equal to one. When the amount of hydrogen in the fuel mixture

Fig. 4 Preferential diffusion coefficient of the progress variable $d_{Y_{p v}}$ (thin lines) and $\lambda / c_{p}$ (thick lines) as a function of reaction progress variable with full line: $X_{\mathrm{H}_{2}}=0.0$, dashed line: $X_{\mathrm{H}_{2}}=0.2$ and dash-dotted line: $X_{\mathrm{H}_{2}}=0.4$

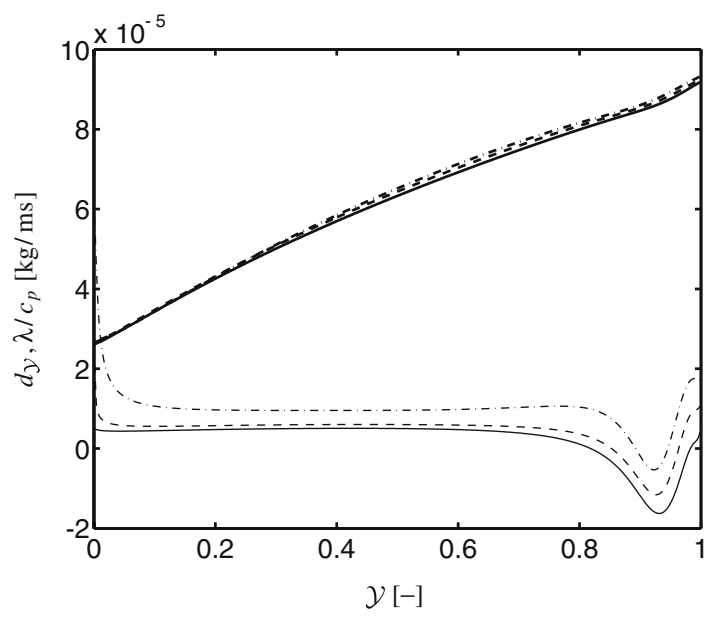


increases the progress variable Lewis number decreases because the Lewis number of hydrogen $\left(L e_{\mathrm{H}_{2}}=0.3\right)$ becomes more important, see the values in the caption of Fig. 3. Also there is a shift of the inner layer position towards the unburnt side. The chemical source term, $\dot{\rho} \mathcal{y}$, the tabulated variable with the largest impact, is shown in the right of Fig. 3. The maximum shifts to the left and it increases with the amount of hydrogen as expected.

In Fig. 4 the non-preferential diffusion coefficient $\left(\lambda / c_{p}\right)$ and the preferential diffusion coefficient $\left(d_{y}\right)$ are plotted as a function of reaction progress variable. The non-preferential diffusion term is larger for all cases, but the preferential diffusion contribution is certainly not negligible. Also, it clearly shows that the preferential diffusion contribution is larger when the amount of hydrogen in the fuel is larger.

As we will see in the course of the paper, application of a one-dimensional manifold will not be sufficient to describe preferential diffusion effects. Therefore we will add a second controlling variable to improve the predictions. The selection of this second controlling variable is described in the next section. However to asses the improvements of results with the dimension increase of the manifold, simulations can be carried out with a one-dimensional manifold. These were performed with the progress variable as described in this section and results are given in Appendix. In this way it was found that for one-dimensional flame propagation without stretch the use of 1D-FGM gives results that can not be distinguished from detailed simulations for all three fuels (therefore these results are not shown in the Appendix). Of course for the case with vortex-flame interactions this is no longer true. The conditions for these simulations are equal to those described in Section 4.1 and the results (with the one-dimensional manifold) are used as reference data in the results section (with the two-dimensional manifold).

\subsection{A second controlling variable}

In de Swart et al. [26], and particularly for hydrogen addition [27], it was shown that when flame stretch and preferential diffusion are present, the enthalpy and the element mass fractions are redistributed in the flame zone. In this section, we will try to capture the changing local conditions by taking into account one extra controlling variable, $Y_{c v 2}$, instead of the complete set of four extra controlling variables. Analysis of 1D flames including hydrogen in the fuel as in [27], supports the assumption that $\Delta h_{i l}$ and $\Delta Z_{j, i l}$ are strongly correlated. Defining the second controlling variable as a combination of elements is convenient since elements do not have a chemical source term. In [27] it was shown that $\Delta Z_{C}$ and $\Delta Z_{H}$ have the largest influence on the mass burning rate due to preferential diffusion. Therefore, the first controlling variable is defined as given in (20) and (21) and the second controlling variable we use here is defined as

$$
Y_{c v 2}=\frac{X_{\mathrm{H}_{2}}^{u}}{0.4} Z_{H}+Z_{C} .
$$

for the cases where hydrogen is present in the fuel (mixtures B and C). This gives a good sensitivity to the hydrogen as desired. For mixture A the second controlling variable is defined as

$$
Y_{c v 2}=Z_{C}+0.01 Z_{O}
$$



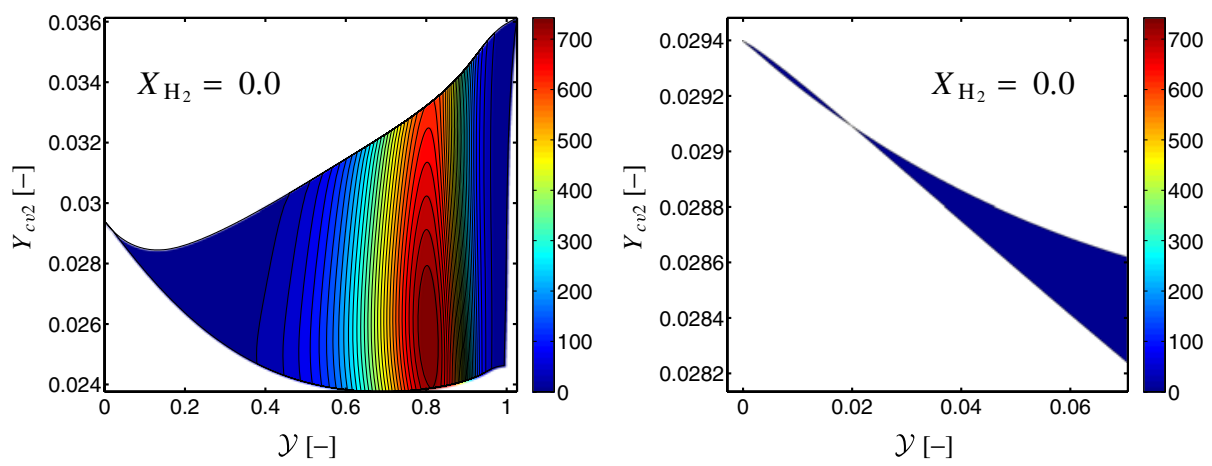

Fig. 5 Chemical source term of $\mathcal{Y}$ as a function of the first two controlling variables with second controlling variable as defined in (22), (left); the right figure shows a zoom

When $Z_{O}$ is not present in the definition of the second controlling variable for mixture A, flamelets are crossing in the manifold and based on the look-up entries more than one value for the variable of interest can be found. This is shown in Fig. 5, where definition (22) is used for mixture A. In the left figure the complete manifold is shown and in the right figure a zoom in the unburnt region of this same manifold is depicted in more detail. It can be observed that individual flamelets cross, which is not allowed.

In Fig. 6 the chemical source terms that are stored in the two-dimensional manifolds are shown as a function of the two controlling variables (21) and (23). It can be seen that every unique combination of $\mathcal{Y}=Y_{c v 1}$ and $Y_{c v 2}$ gives a single value for the chemical source term. In this way a convenient set of controlling variables is defined.

\section{Flame-Vortex Interaction}

\subsection{Problem setup}

In order to study the interaction of eddies with flames, the initially flat flame will be subjected to vortices that enter the domain with a certain background velocity. Two-dimensional divergence-free vortical flow is prescribed according to

$$
\begin{gathered}
u(x, y)=A_{w} \sin \left(2 \pi k_{0} x\right) \cos \left(2 \pi k_{0} y\right), \\
v(x, y)=-A_{w} \cos \left(2 \pi k_{0} x\right) \sin \left(2 \pi k_{0} y\right),
\end{gathered}
$$

and is convected into the domain of size $[\mathrm{x}, \mathrm{y}]=\left[\begin{array}{ll}0 & L_{x}\end{array}\right]\left[\begin{array}{ll}0 & L_{y}=L_{x} / 2\end{array}\right]$ with a speed $u_{i n}$. The domain length $L_{x}$ is $8 \mathrm{~mm}$ long and the domain is periodic in $y$ direction. The initial flame is positioned at half the length and the outflow is non-reflective. Parameters $A_{w}$ and $k_{0}$ are used to set the vortex amplitude and the wave length and these are equal to $A_{w}=0.6 \mathrm{~m} / \mathrm{s}$ and the wave number matches the domain width, $k_{0}=2501 / \mathrm{m}$. The convecting velocity is set equal to the laminar burning velocity for 
Fig. 6 Chemical source term of $\mathcal{Y}$ as a function of the first two controlling variables $\mathcal{Y}$ and $Y_{c v 2}$
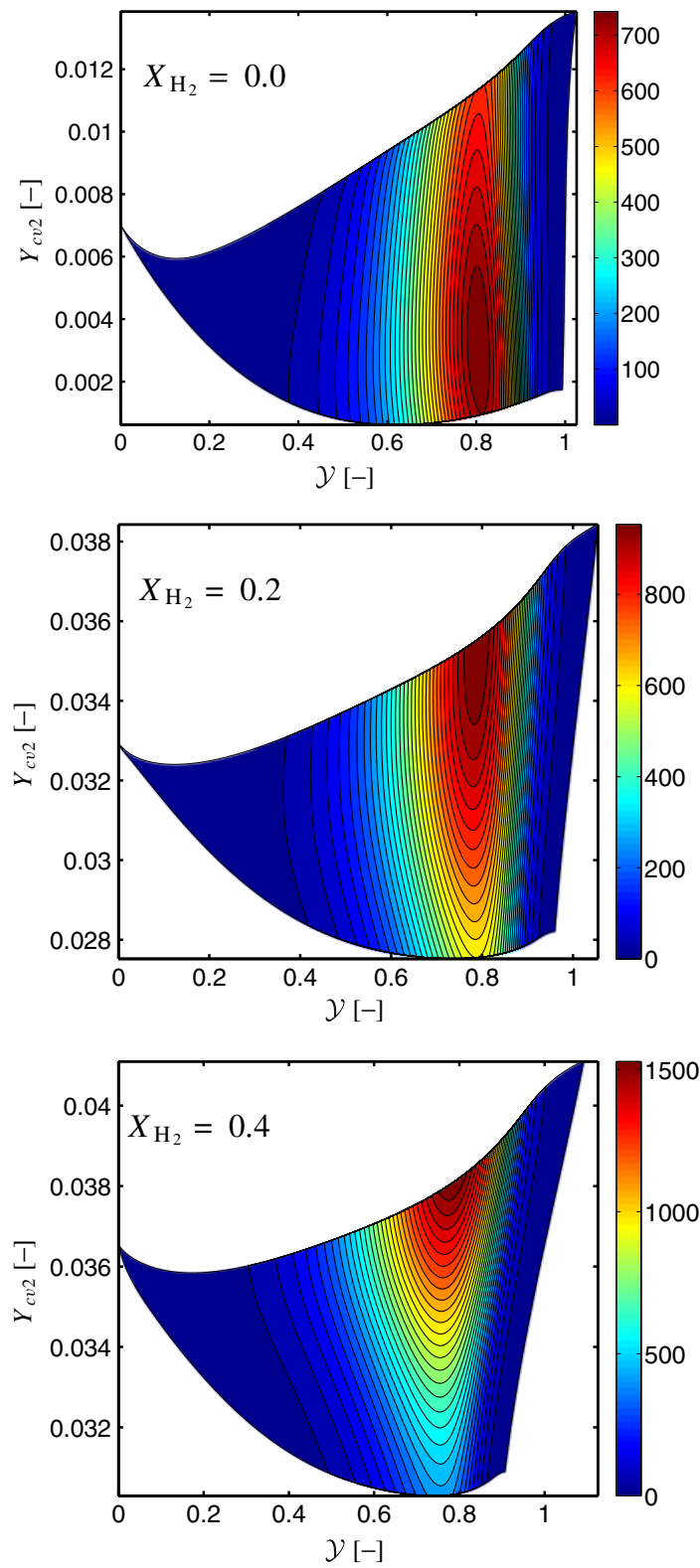

each case $u_{\text {in }}=s_{L}^{0}$. For a pure methane flame this is $u_{\text {in }}=0.18 \mathrm{~m} / \mathrm{s}$. The decay of the prescribed flow is known analytically,

$$
u_{r m s}(t)=\sqrt{A_{w}^{2} / 2} \exp \left(-2 v\left(2 \pi k_{0}\right)^{2} t\right),
$$

which depends on the initial intensity (which is equal to $\sqrt{A_{w}^{2} / 2}$ ), the viscosity $(v)$, the wave number of the vortex $\left(k_{0}\right)$ and time $(t)$. Also the intensity as function of 
Fig. 7 Computational domain of the two-dimensional flame-vortex simulations

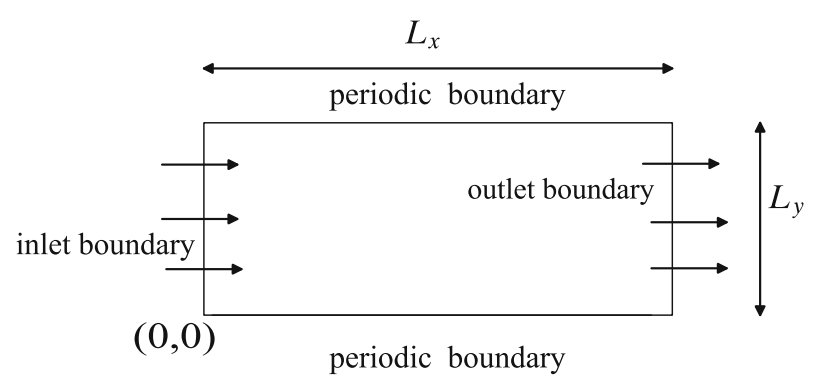

distance from the inflow boundary can be determined by converting time to distance with the background convection velocity.

The computational domain is two-dimensional as shown in Fig. 7. The grids used in this case consists of $128 \times 64$ points and time steps of $\Delta t$ equal to $2.5 \cdot 10^{-8}$ seconds are taken. The time can be scaled with a vortex time, defined as

$$
t_{v}=\frac{1}{2 k_{0} u_{i n}} .
$$

The flame surface area and the integral consumption rate are key parameters in the analysis of the global combustion characterization of the flow-flame interactions. Different definitions for the flame surface area can be used, in this work an inner reaction layer surface area is used,

$$
A_{f, i l}=\int_{V} \delta\left(\mathcal{Y}-\mathcal{Y}_{i l}\right) \mathrm{d} V
$$

as well as the integral flame surface area,

$$
A_{f, i n t}=\int_{V}|\nabla \mathcal{Y}| \mathrm{d} V
$$

The flame surface, $A_{f, i l}$, is evaluated with an integration along isocontours determined in postprocessing with Matlab (of course in the 2D situation the area is an area per unit length in the out-of-plane direction, the same holds for the volume). The burning rate, $m_{T}$, is defined by the surface integration of the source term of the progress variable over the domain. From the definition of the integral flame surface area, (29), it becomes clear that preheat zone deformation has a contribution in the integral flame surface area $A_{f, i n t}$. It is possible that vortices penetrate the preheat zone but do not reach the reaction zone (which is located towards the burnt side of the flame). In this case the chemical processes are not influenced by the flow but an increase in integral flame surface area $A_{f, \text { int }}$ is seen. It is expected that when flow structures are relatively large compared to the flame thickness $\delta_{f}$, the inner layer flame surface area will be (approximately) equal to the integral flame surface area, $A_{f, i l} \approx A_{f, i n t}$. However, when the flow scales become relatively small, the integral flame surface area will become larger than the inner layer flame surface area, $A_{f, i n t}>A_{f, i l}$. In this case it is expected that, since the chemical reactions take place in a relatively thin layer positioned near the burnt side of the flame, that the mass consumption is proportional to the inner layer flame surface area $A_{f, i l}$, the integral flame surface area $A_{f, \text { int }}$ is then expected to have a weaker correlation with the mass consumption. 


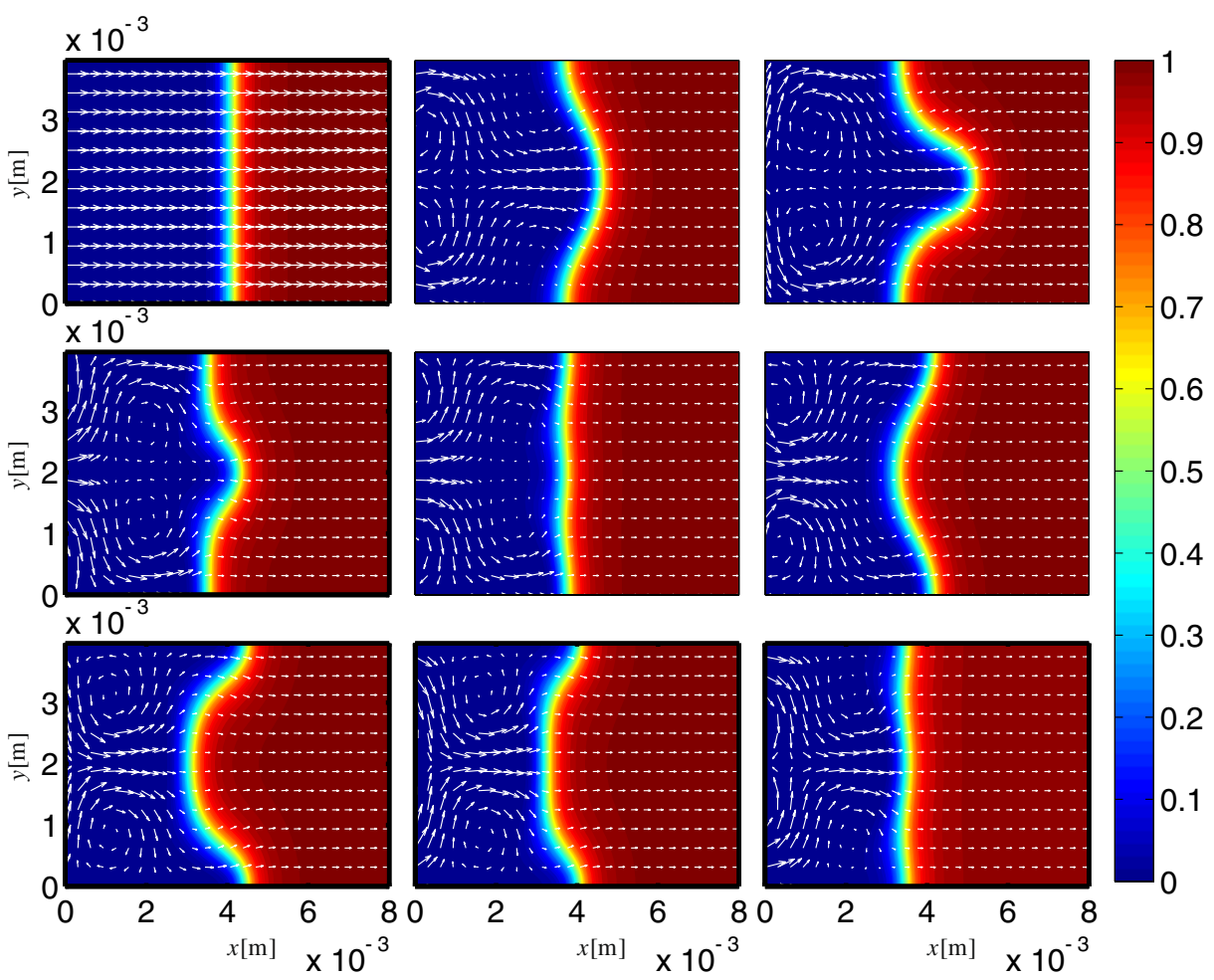

Fig. 8 Development of the premixed flame under the influence of vortical flow with reaction progress variable in color and density-weighed velocity in white arrows. From left to right and from top to bottom these figures show $t=0, t=1.6 t_{v}, t=2.0 t_{v}, t=2.3 t_{v}, t=2.5 t_{v}, t=2.7 t_{v}, t=3.0 t_{v}$, $t=3.2 t_{v}$ and $t=3.4 t_{v}$

\subsection{Results}

A typical cycle of flame behavior is displayed in Fig. 8. This is the result of a simulation for pure methane where the reaction progress variable field is displayed at different times. It can be seen that the vortex-pairs that are fed through the inlet cause the flame to move back and forth. Also it can be seen that the flow in the burnt region is directed towards the outlet and the vortices have almost disappeared. This is mainly due to the expansion over the flame. The density decrease results in increasing velocities.

The inner layer surface area and the turbulent burning rate are plotted in Fig. 9 as a function of (dimensionless) time. The turbulent burning rate is defined by $m_{T}=\int_{V} \dot{\rho}_{Y} d V / A^{0}$, with $A^{0}$ the area of the cross section of the domain. Differences between the cases exist for flame surface area of the inner layer as well as for integral consumption. Cases of vortex-flame interactions are indicated with Case-v, supplemented with a letter, indicating the mixture. Case-v-A has the lowest peaks and returns to an almost flat flame configuration in between vortices. Increasing the hydrogen content in the fuel (i.e. Case-v-B and Case-v-C) shows larger peak values and also no intermediate flat configuration is found. Although not very pronounced, this is indicative for an instability that occurs when adding hydrogen. 

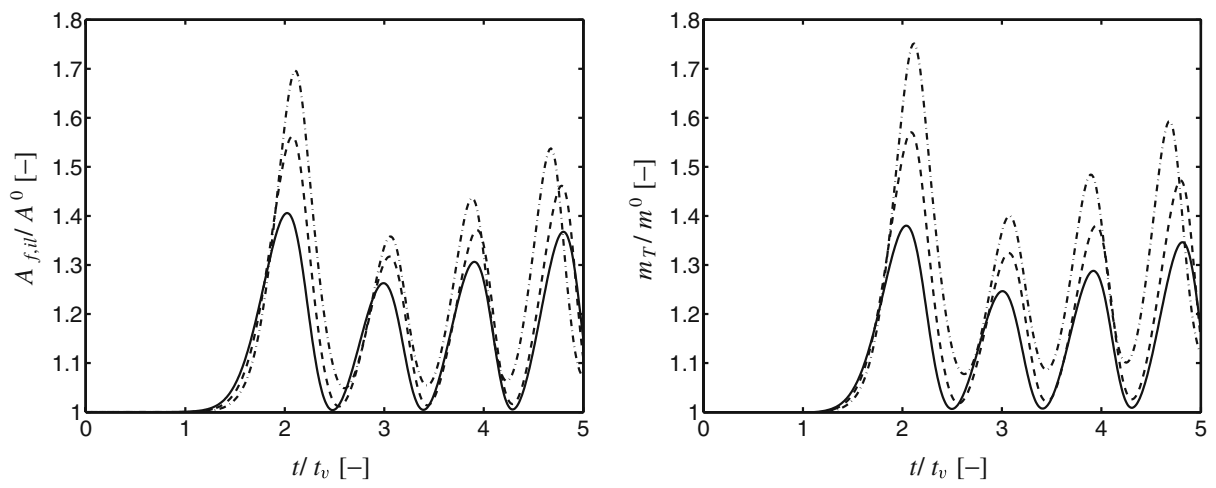

Fig. 9 Flame surface area of reaction layer (left) and mass consumption (right) for a SENGA-FGM simulation with full line: Case-v-A, dashed line: Case-v-B and dash-dotted line: Case-v-C

Probably this corresponds to the formation of cellular structures. However we did not perform calculations to prove this behavior because here we are interested in the computational approach rather then the physical phenomena. Therefore the conditions of the flow-flame interaction are kept at relatively short integration times, such that the change in flame surface is limited (when the flame propagates to the inflow the vortices have decayed less and therefore they are more intense). This results in a limited complexity and associated resolution such that we can accurately defend the modeling approach.

There are relatively large differences between the current results and the results as obtained with the one-dimensional manifold, (Fig. 27). Even the trend in both the flame surface area as well as the integral consumption rate is opposite to each other. With the 2D-FGM an enhanced flame surface area increase and mass consumption appears. Also there is an advancement of the phenomena, in contrast to 1D-FGM results where there is a delay with higher amounts of hydrogen. In order to show what happens in the simulation with the 2D-FGM, in Fig. 10 the iso-surface of the
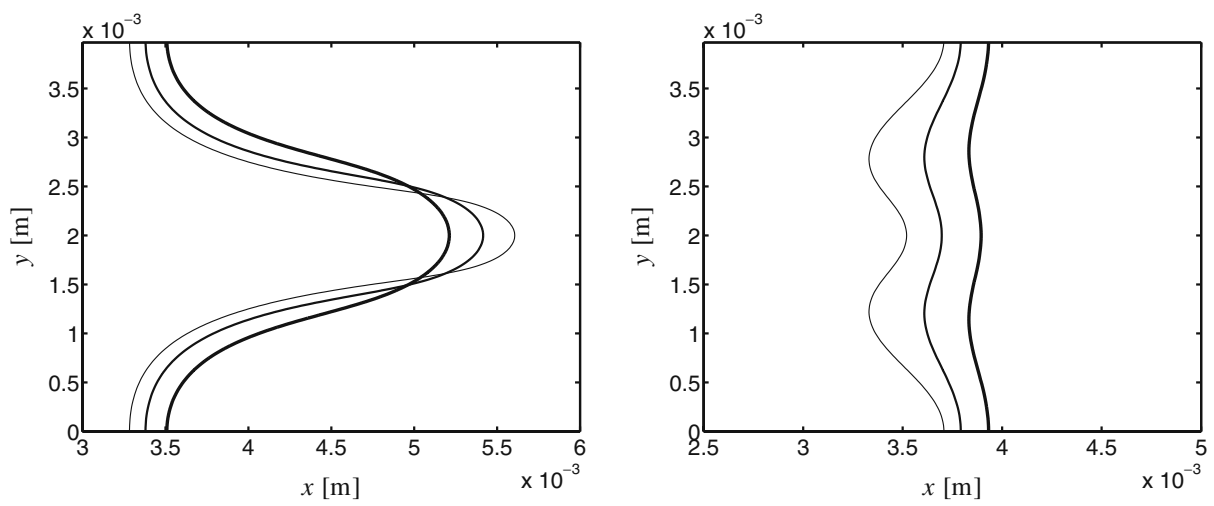

Fig. 10 Iso-contour of inner layer progress variable at the first peak (left figure) and at the lowest value between the first and second peak (right figure), with thickest line: Case-v-A, medium line: Case-v-B and thinnest line: Case-v-C 

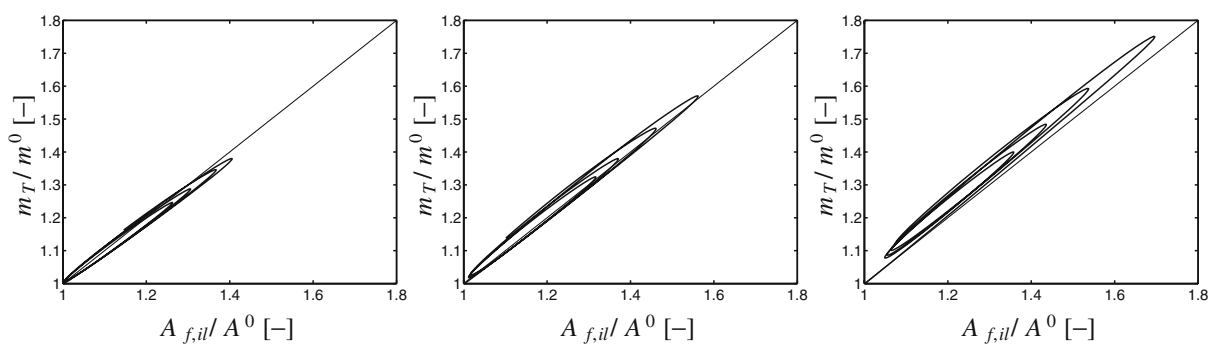

Fig. 11 Mass consumption versus flame surface area of reaction layer with left: Case-v-A, middle: Case-v-B and right: Case-v-C

inner layer is plotted for all three cases both at the first peak and at the lowest value of flame surface between the first and second peak.

The flame surface area is subsequently plotted against the integral consumption in Fig. 11. All cases show an ellipsoidal behaviour where Case-v-C has the widest and Case-v-A has the narrowest lateral extent. According to the theory, an infinitely thin reaction layer, with Lewis numbers equal to one, should show realizations positioned at a line with a slope equal to one. The differences between the results of different fuels are larger with the 2D manifold than when a 1D-FGM is used (as can be observed in Fig. 30 in the Appendix). Especially the range of values of the total consumption and flame surface density is almost constant for 1D-FGM, going from 1 to about 1.45 for all cases, whereas in the 2D-FGM the maximum increases from 1.4 to 1.6 and 1.7 for the increase in hydrogen. Also the minimum values is lifted from unity.

At times I: $t / t_{v}=1.9$, II: $t / t_{v}=2.3$, III: $t / t_{v}=3.2$ and IV: $t / t_{v}=3.9$ flamelets are tracked and from the relation

$$
\frac{m_{i l}}{m_{i l}^{0}}=1-\mathcal{M} \frac{K a_{\mathcal{Y}, i l}}{L e_{\mathcal{Y}}},
$$

the Markstein numbers were determined. The flamelet tracking is performed, as in [18]. By taking initial points close to the flame surface we follow flamelet structures in the direction of the fastest increase of progress variable. With the data obtained we determine the Karlovitz number by (2) and the local mass burning rate by

$$
m_{i l}=\left.\frac{\dot{\rho} \mathcal{Y}+\nabla \cdot\left(\rho D_{\mathcal{Y}} \nabla \mathcal{Y}\right)}{|\nabla \mathcal{Y}|}\right|_{i l} .
$$

Figure 12 shows the relation between the scaled inner layer mass burning rate and the scaled Karlovitz integral. Each dot in this scatterplot represents an entire flamelet. For all cases and times almost linear profiles are found. Note that the distributions are not symmetric in positive and negative stretch. However when assessing mean Karlovitz integrals and mean inner layer mass burning rates (Table 2), it is found that there is approximately as much positive stretch as there is negative stretch. 

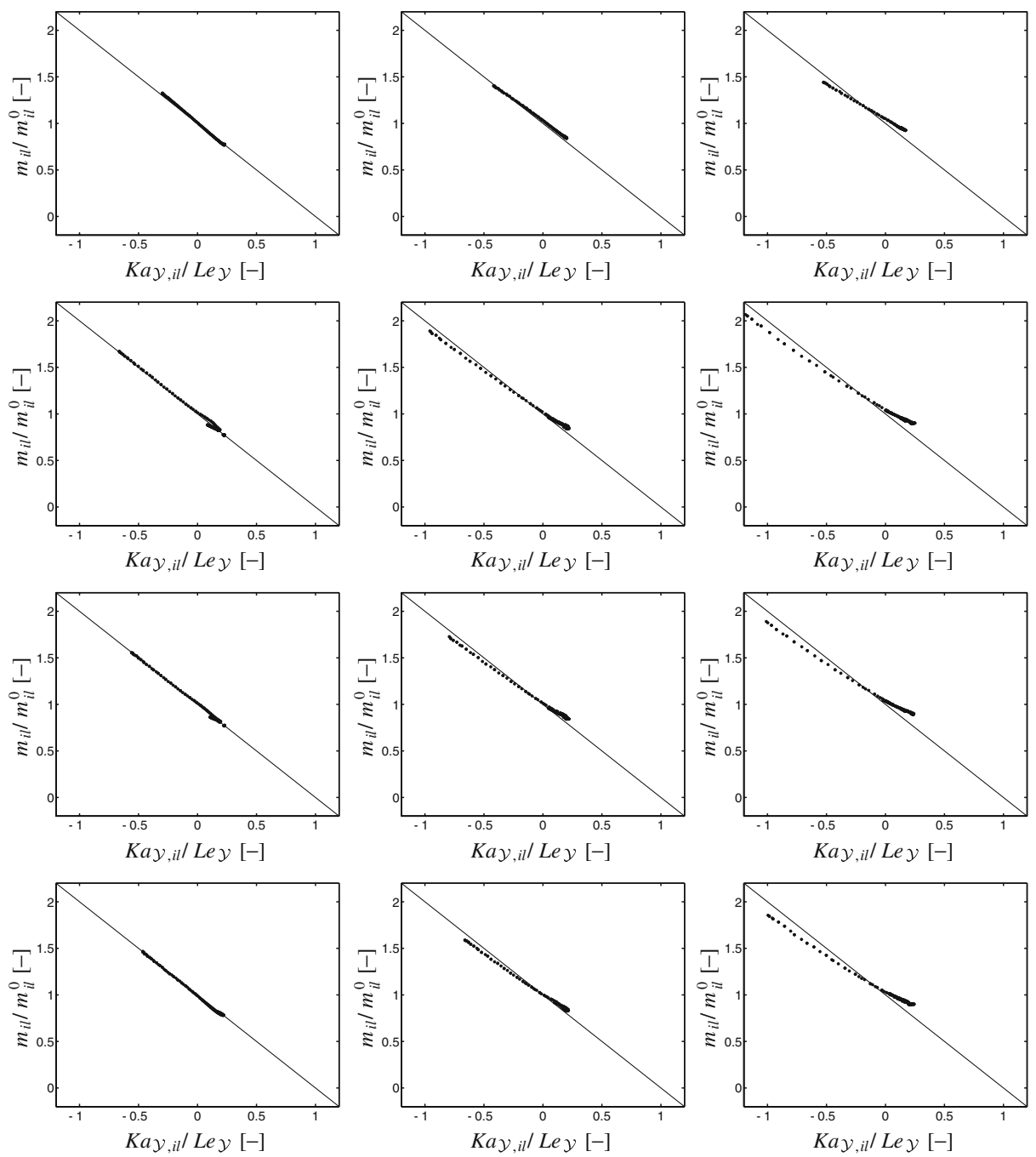

Fig. 12 Scaled inner layer consumption as a function of Karlovitz integral for (left to right) Case-v-A, Case-v-B and Case-v-C at (top to bottom) time I to IV

Additionally, in Table 2, the slopes of the profiles are presented. These slopes were found by performing a least squares linear fit through all data-points. When there is no hydrogen present in the fuel a Markstein number of approximately one is found. When hydrogen is added the Markstein number decreases, $\mathcal{M} \approx 0.87$ for $X_{\mathrm{H}_{2}}=0.2$ and $\mathcal{M} \approx 0.75$ for $X_{\mathrm{H}_{2}}=0.4$.

This clearly indicates a stronger preferential diffusion effect in these stretched flames than was seen in the simulations where a 1D-FGM was used. By adding a manifold dimension we are able to capture a stronger preferential diffusion effect. However, the Markstein numbers clearly differ from the Markstein numbers found 
Table 2 Mean values of Karlovitz integral, scaled inner layer mass consumption and $\mathcal{M}$ of the three cases at different times

\begin{tabular}{|c|c|c|c|}
\hline & Case-v-A & Case-v-B & Case-v-C \\
\hline \multicolumn{4}{|l|}{ Time I } \\
\hline$<K a_{\mathcal{Y}, i l} / L e y>$ & 0.05 & 0.05 & 0.06 \\
\hline$<m_{i l} / m_{i l}^{0}>$ & 0.95 & 0.98 & 1.00 \\
\hline $\mathcal{M}$ & 1.05 & 0.88 & 0.67 \\
\hline \multicolumn{4}{|l|}{ Time II } \\
\hline$<K a_{\mathcal{Y}, i l} / L e_{\mathcal{Y}}>$ & -0.01 & 0.00 & 0.01 \\
\hline$<m_{i l} / m_{i l}^{0}>$ & 1.01 & 1.02 & 1.06 \\
\hline $\mathcal{M}$ & 1.01 & 0.88 & 0.80 \\
\hline \multicolumn{4}{|l|}{ Time III } \\
\hline$<K a_{\mathcal{Y}, i l} / L e y>$ & 0.01 & 0.02 & 0.02 \\
\hline$<m_{i l} / m_{i l}^{0}>$ & 0.98 & 1.00 & 1.04 \\
\hline $\mathcal{M}$ & 1.00 & 0.86 & 0.76 \\
\hline \multicolumn{4}{|l|}{ Time IV } \\
\hline$<K a y, i l / L e y>$ & 0.03 & 0.03 & 0.02 \\
\hline$<m_{i l} / m_{i l}^{0}>$ & 0.96 & 0.98 & 1.03 \\
\hline $\mathcal{M}$ & 1.01 & 0.85 & 0.76 \\
\hline
\end{tabular}

for the one-dimensional stretched flames with detailed chemistry in Section 2. In the next section, the differences between the values of the Markstein numbers will be explained.

\subsection{Detailed flamelet analysis of the results}

\subsubsection{Convergence with manifold dimension}

With flamelet analysis, as defined and performed in [18], we can analyse and compare flamelet profiles. In such an analysis we track one-dimensional flamelets in an instantaneous simulation result. The (one-dimensional) flame stretch and curvature fields from the flamelets, which are extracted from the two-dimensional simulations, are imported in CHEM1D, which solves one-dimensional flamelets using the supplied curvature and stretch fields. These one-dimensional flamelets can then be solved with any manifold, but also with detailed chemistry. In this way we can test the flamelet approach by using the same manifold as in the actual simulations. On the other hand we can also investigate the convergence of manifolds with higher dimensions under realistic stretch and curvature conditions. The convergence that we will look at is between a 1D-FGM manifold, a 2D-FGM manifold and detailed chemistry. In this way we can quantitatively evaluate the improvement of the application of a $2 \mathrm{D}$ FGM manifold. Note that when there is no flame stretch and curvature a constant mass burning rate is found. However, when a flame stretch and curvature field is prescribed, this yields a mass burning rate as a function of position.

Thus, it is expected that a 2D-FGM simulation gives more accurate results than a 1D-FGM simulation. In this section, a direct comparison is made between three types of chemistry, using flame stretch and curvature profiles from the two-dimensional flame-vortex simulations:

1. CHEM1D-detailed chemistry: In this simulation a detailed chemical mechanism is used (GRI3.0). This resulting flame solution is used as a benchmark. 
Fig. 13 Typical flamelets used for validation from Case-v-C at time III

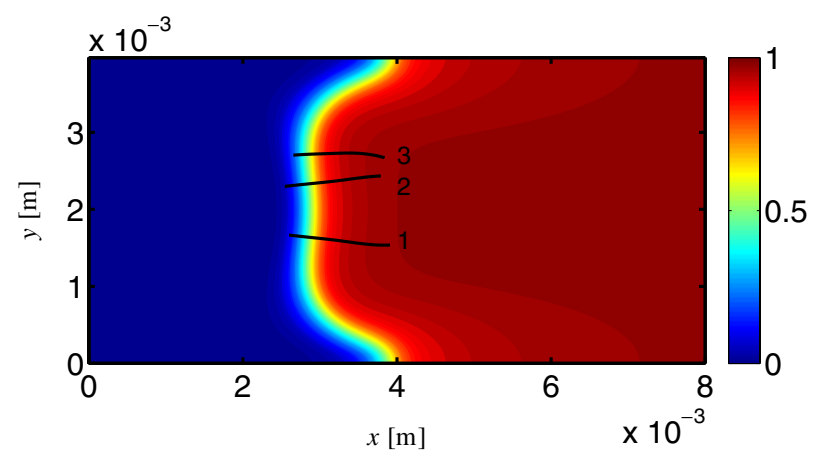

2. CHEM1D-2D-FGM: Here a 2D-FGM is used in postprocessing of the SENGA results.

3. CHEM1D-1D-FGM: Using a 1D-FGM, results will be compared to the two previous simulations.

In the flame solution of Case-v-C at time III, three flamelets are chosen (see Fig. 13) and the flame stretch fields of these flamelets are used as input in CHEM1D. The results for the two controlling variables and the mass burning rate are shown in Figs. 14 and 15 for two flamelets showing different realizations. Flamelet 2 behaves quite similar to flamelet 1 , this flamelet will be used in another analysis in the next section.

Both controlling variables are displayed, it is shown that for the first controlling variable $Y_{c v 1}$ the simulations show approximately the same solution for all simulation
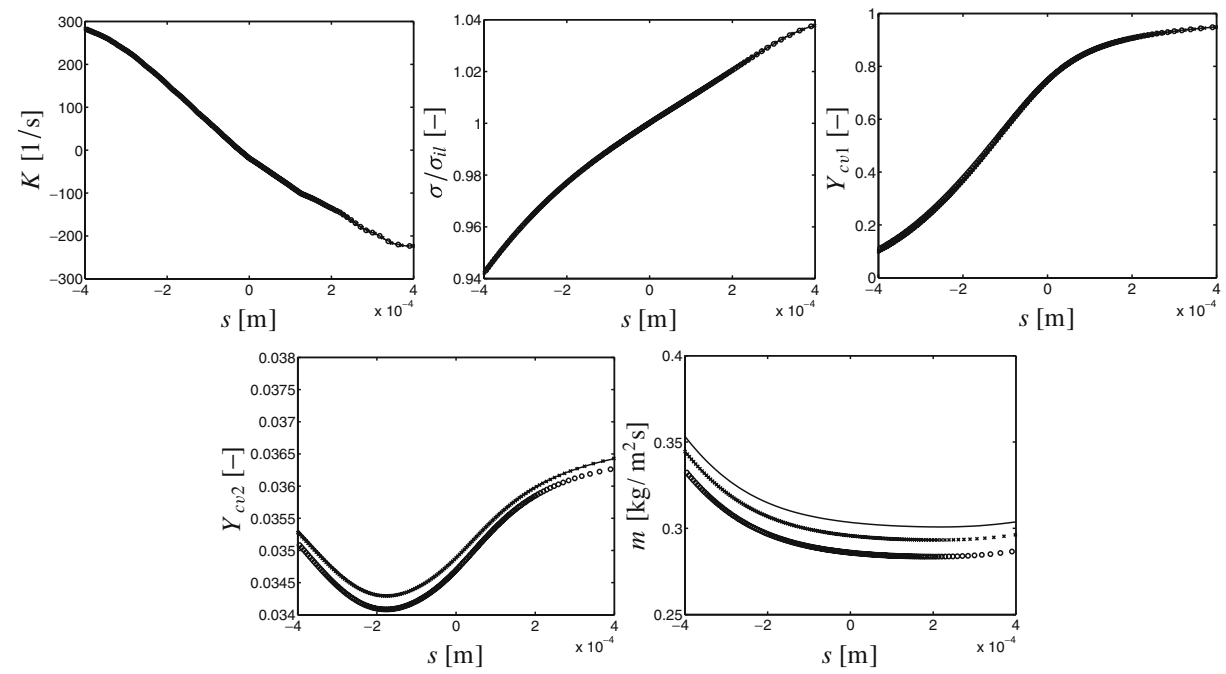

Fig. 14 Flame stretch rate $K$ (top left), curvature $\sigma$ (top middle), progress variable $Y_{c v 1}$ (top right) and second controlling variable $Y_{c v 2}$ (bottom left) and mass burning rate $m$ (bottom right) of flamelet 1 extracted from Case-v-C (with laminar flame time $\tau=2[\mathrm{~ms}] ; 1 / \tau=500[1 / \mathrm{s}]$ ) at time III. Full line: detailed chemistry, circles: 1D-FGM and crosses: 2D-FGM 


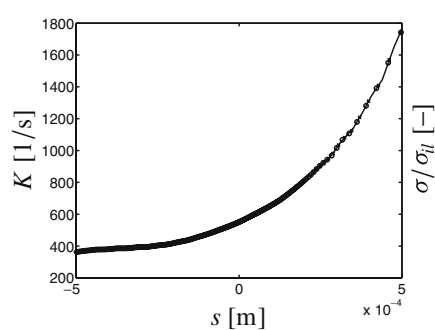

$s[\mathrm{~m}]$
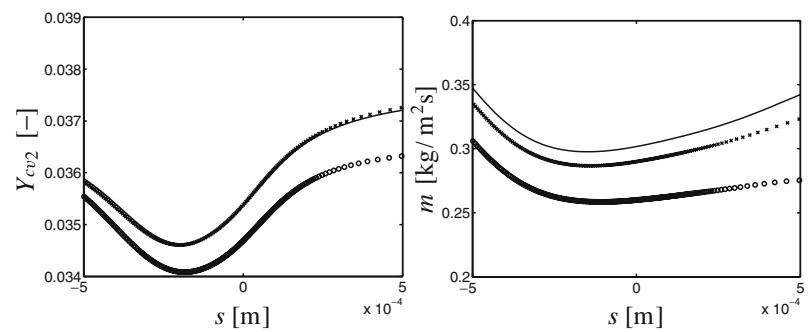

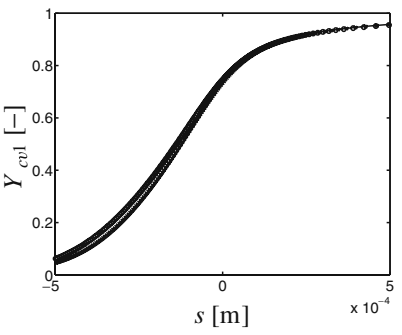

Fig. 15 Same as previous figure but for flamelet 3

techniques, while for the second controlling variable, $Y_{c v 2}$, differences appear. Moreover, it is found that the profiles for $Y_{c v 1}$ and $Y_{c v 2}$ in the 2D-FGM is closer to the detailed chemistry solution than in the 1D-FGM. Looking at the mass burning rate, for all three flamelets shown, the mass burning rate of the 2D-FGM is closer to that of the detailed case than the mass burning rate of the 1D-FGM. It can be concluded that adding a dimension to the flamelet database (i.e. moving from a 1D-FGM to a 2D-FGM) results in an increase in the accuracy of the solution of both controlling variables and the mass burning rate. It is also seen that the 2D-FGM can reproduce almost the exact same results as the detailed chemistry simulation (1D stationary, but stretched) for the second controlling variable $Y_{c v 2}$. Still, the mass burning rate in a $2 \mathrm{D}-\mathrm{FGM}$ does not exactly coincide with the detailed chemistry solution. The evaluation of the 1D-FGM in this test can be regarded as an a priori test, whereas the actual simulation with a $1 \mathrm{D}-\mathrm{FGM}$ as given in the Appendix, is an a posteriori test. The a priori and a posteriori tests are consistent in the outcome that 1D-FGM predicts a less accurate burning velocity.

\subsubsection{Flamelet and numerical accuracy}

In the previous analysis it was assumed that the stretch and curvature field are accurate and that the flamelet hypothesis holds. However a number of causes for differences should be analyzed as well:

1. The modeling error. This indicates the correctness of the flamelet approximation. Besides the current truncation of the number of controlling variables (treated in the previous section) also unsteady and tangential transport effects are responsible. The latter two effects are clearly described and explained in e.g. [17].

2. The one-dimensional flame stretch and curvature profiles have to be interpolated on the grid used in CHEM1D, giving possible errors.

3. Different discretization methods are used in SENGA and in CHEM1D. This can cause small differences in the flame solution. 

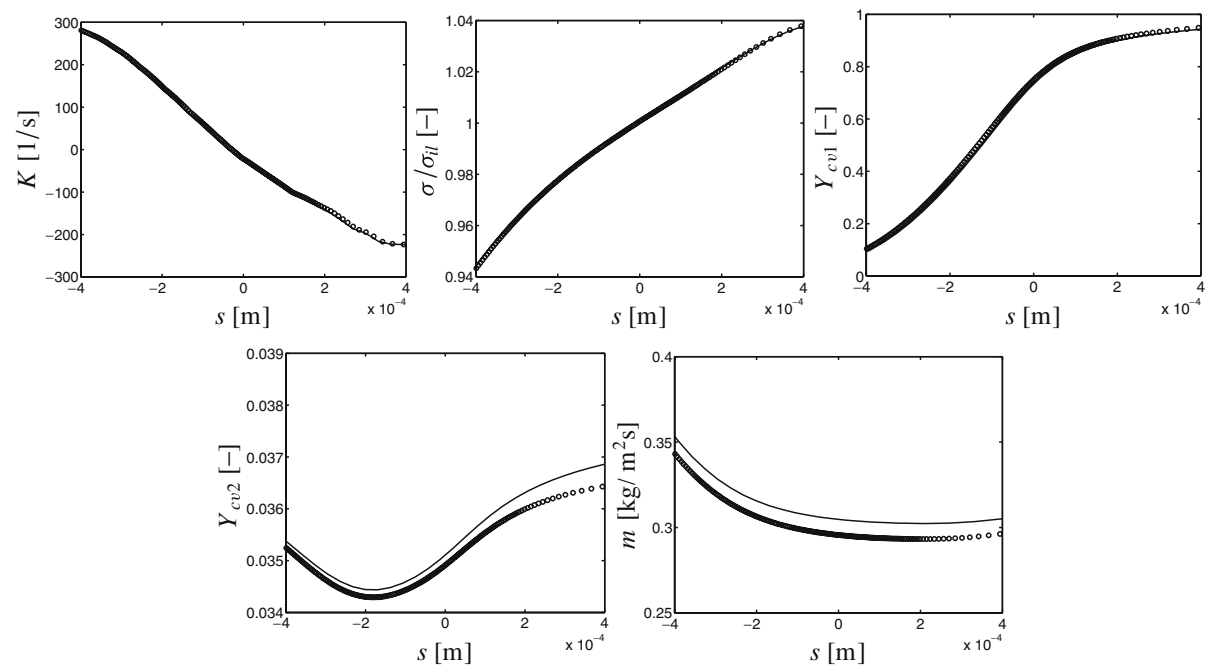

Fig. 16 Flame stretch rate $K$ (top left), curvature $\sigma$ (top middle), progress variable $Y_{c v 1}$ (top right) and second controlling variable $Y_{c v 2}$ (bottom left) and mass burning rate $m$ (bottom right) of flamelet 1 extracted from Case-v-C at time III. Full line: SENGA-2D-FGM and circles: CHEM1D-2D-FGM

The differences due to these items can be evaluated by comparing the result of the 2D-FGM in CHEM1D of the previous section with the actual SENGA result. By doing this the implementation of the 2D-FGM method in SENGA is validated. For clearness: in both simulations the same 2D-FGM database is used. Of course also a 1D-FGM can be used in these a priori CHEM1D calculations. The same flamelets are chosen as in the previous section. The results for the two controlling variables and the mass burning rate are shown in Figs. 16 and 17. The solution
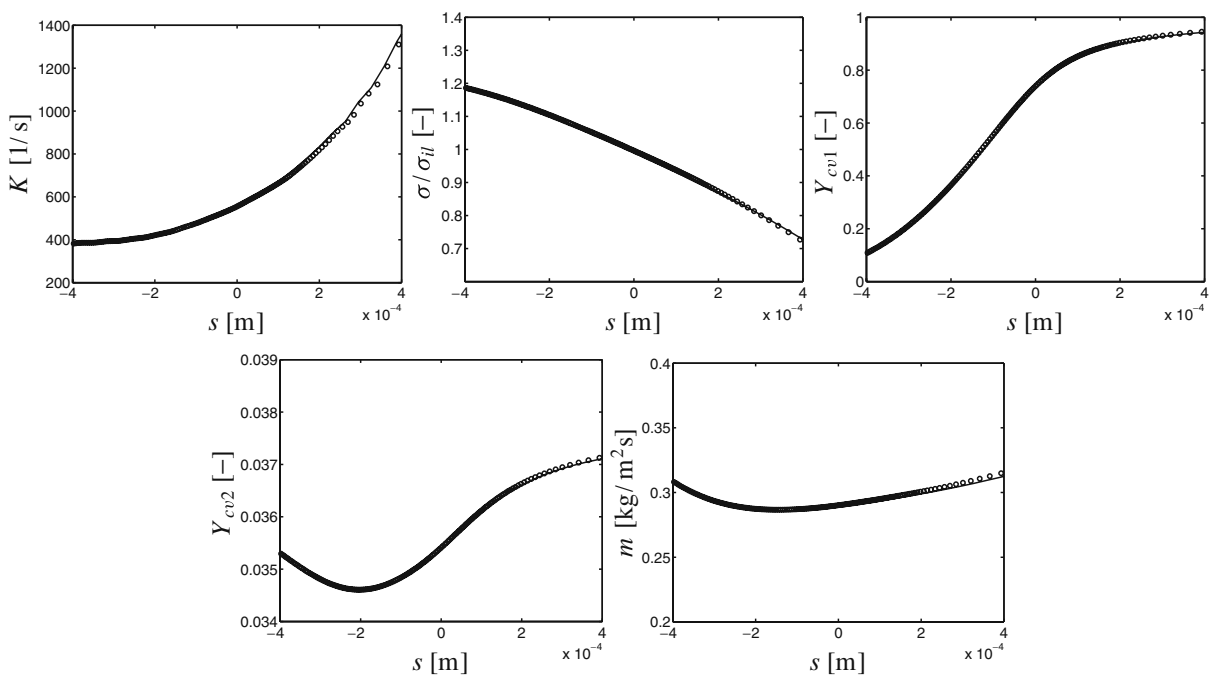

Fig. 17 Same as previous figure but for flamelet 3 
for the first controlling variable is very accurate for both flamelets, indicating that errors related to interpolation and meshing (2 and 3) are negligible. It is expected that the contributions that arise when tangential transport becomes important, are small compared to stretch terms in most circumstances, although this might not be justified under extreme circumstances, such as encountered in laminar thermodiffusive unstable hydrogen flames or near local flame quenching.

From the previous paragraph we conclude that the cause for significant differences can only be due to the unsteady and tangential terms. From the differences in the solution for the second controlling variable in Figs. 16 and 17, it can be seen that these terms can cause small deviations between the CHEM1D-2D-FGM solution and the SENGA-2D-FGM solution as in flamelet 1 . So for the current conditions unsteady and tangential flamelet errors and the error due to the truncation of the number of controlling variables are similar in magnitude. Therefore adding more controlling variables would not improve the quality of the simulations significantly.

\subsubsection{Enthalpy and element variations}

Now, it is interesting to investigate the relation between the second controlling variable and the local changes in enthalpy and element mass fraction. In order to explicitly show the relation, we can plot the change in second controlling variable $\Delta Y_{c v 2}$ versus the different changes in elements $\Delta Z_{j}$ and the change in enthalpy $\Delta h$. In Fig. 18, the coupling between $\Delta Y_{c v 2}$ and $\Delta Z_{j}, \Delta h$ in a detailed chemistry simulation with flame stretch and curvature extracted from a SENGA-simulation is compared to the coupling between $\Delta Y_{c v 2}$ and $\Delta Z_{j}, \Delta h$ that is stored in the flamelet. The difference between these two simulations must originate from the flame stretch and curvature field. The flame solutions stored in the manifold are constructed using detailed chemistry (as well), but instead of flame stretch and curvature from a SENGA-simulation, a constant curvature (with corresponding flame stretch rate, see Section 3.3) is used.

It is found that the coupling between the element changes $\Delta Z_{j}$ and the second controlling variable is relatively accurately reproduced by the $2 \mathrm{D}-\mathrm{FGM}$. However in the (detailed) simulation with the stretch field from SENGA, the enthalpy change,

Fig. 18 Changes in element mass fraction $\Delta Z_{j}$ and changes in enthalpy $\Delta h$ as a function of the second controlling variable $Y_{c v 2}$ for data stored in flamelet (full lines) and the three extracted flamelets. Full circles: indicate contribution of hydrogen, stars indicate that of carbon, enthalpy is indicated by triangles and the contribution of oxygen is indicated by squares. Numbers 1, 2 and 3 correspond to flamelet 1,2 and 3

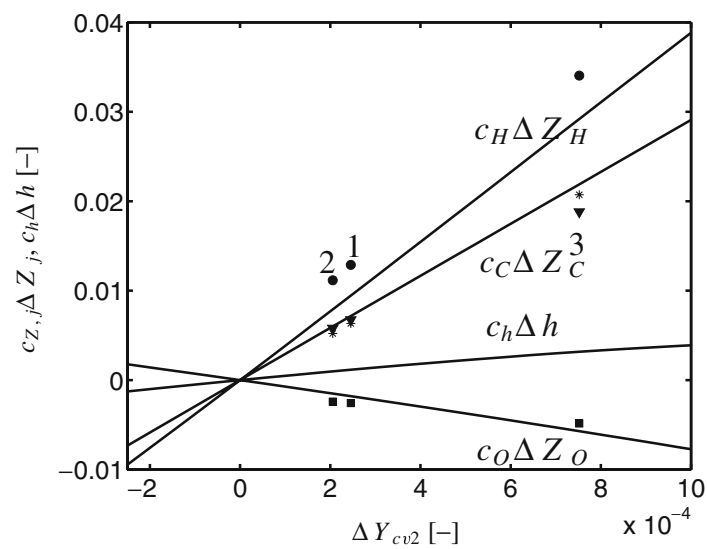


Fig. 19 Heat release at the inner layer position $\omega_{T, i l}$ as a function of second controlling variable $Y_{c v 2}$ for data stored in flamelet (full line) and for the three extracted flamelets (numbered squares)

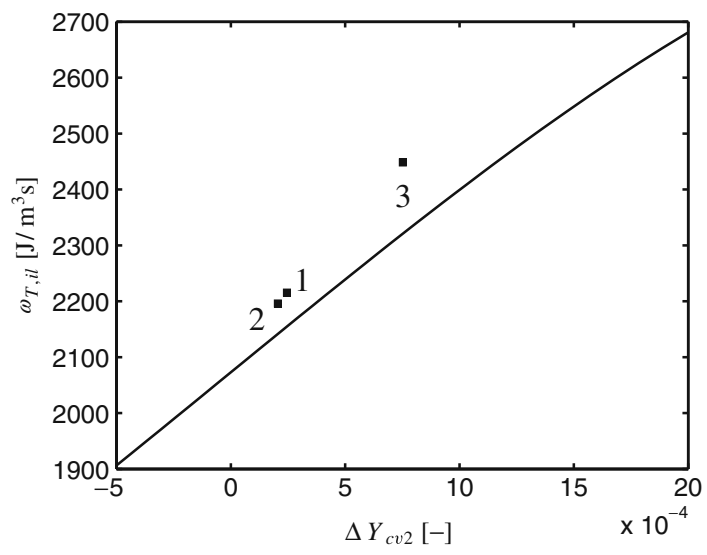

$\Delta h$, is much larger than was expected (line with lowest slope) from the coupling stored in the flamelet. This inaccuracy has an effect on the resulting mass burning rate. This is observed in Fig. 19, where the heat release at the inner layer position (which is a measure for the chemical source term) is shown as a function of the change in second controlling variable $Y_{c v 2}$. For both simulations that were used to construct the manifold as well as for the simulation using the stretch and curvature field from SENGA, the relation is shown. It can be seen that the heat release is larger in the simulations with the flame stretch and curvature field from SENGA (squares 1 to 3). This is consistent with the observation in Fig. 18, where a larger enthalpy was seen.

Concluding, it was shown that a 2D-FGM is more accurate than a 1D-FGM and that results using a 2D-FGM are close to a detailed solution. Assuming a coupling between the changes in element mass fractions $\Delta Z_{j}$ and $\Delta h$, the local change in stoichiometry and enthalpy is taken into account by using a second controlling variable. It is also shown that this second controlling variable does not always capture the full effect of local changes in elements and enthalpy. The small inaccuracy that was introduced by taking only one extra controlling variable $Y_{c v 2}$, results in a different heat release and this has an effect on the mass burning rate. It seems that an increasing dimension of the manifold will not improve the approximation significantly.

\section{Turbulent Flames}

\subsection{Results of the 2D FGM aproach}

The 2D-FGMs are also used in the simulation of turbulent flames. Since in most practical applications turbulent conditions appear, it is useful to investigate whether the numerical code can capture preferential diffusion effects when a broader range of length scales and flow intensities is present. Again three mixtures are simulated, where the mole fraction of hydrogen in the fuel is $X_{\mathrm{H}_{2}}=0.0$ (Case-t-A), $X_{\mathrm{H}_{2}}=0.2$ (Case-t-B) and $X_{\mathrm{H}_{2}}=0.4$ (Case-t-C), respectively. Instead of the vortices here a 
Fig. 20 Flame solutions of turbulent SENGA-FGM flame (top: Case-t-A, middle: Case-t-B and bottom:

Case-t-C), where the colored iso-contours: indicate temperature and the arrows indicate flow in the plane where $z=0$ at time $t=3.4 t_{t}$
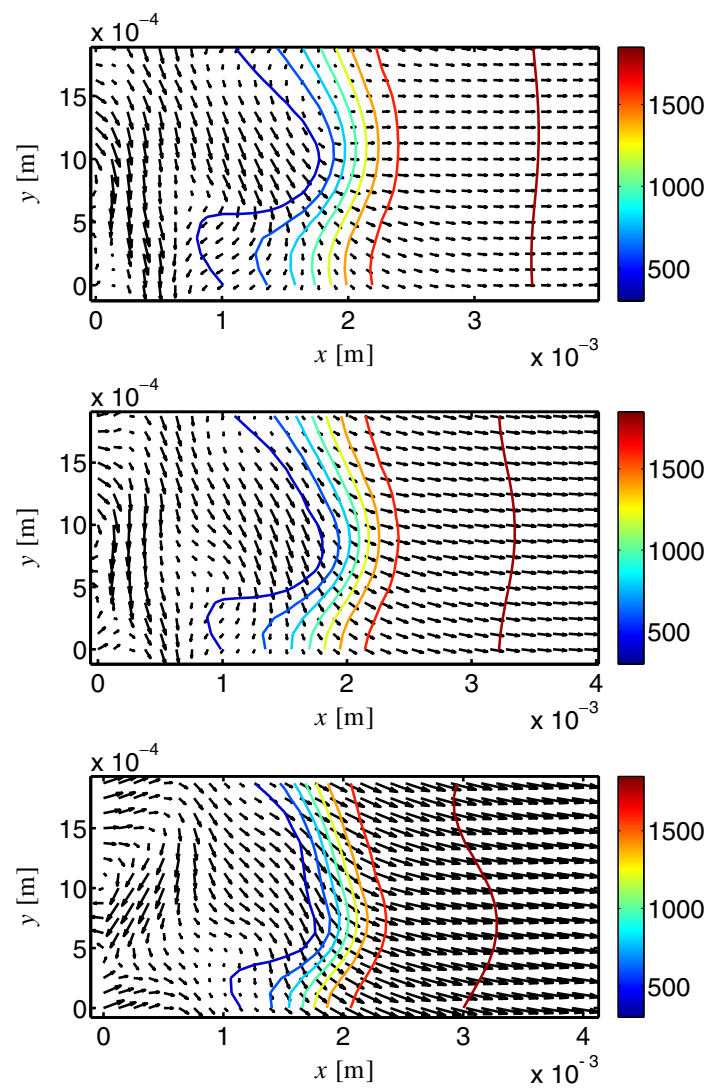

turbulent field is convected in the domain. Now the simulations are threedimensional on a $128 \times 64 \times 64$ grid and the field is initialized with a BatchelorTownsend spectrum on a 64 cube grid with rms velocity equal to $1.55 \mathrm{~m} / \mathrm{s}$, a longitudinal integral length scale of $L_{f}=0.664 \mathrm{~mm}$ and a Kolmogorov length scale of $\eta=0.039 \mathrm{~mm}$. When the turbulence arrives at the flame the Kolmogorov scale is increased, thus remaining in the thin reaction zones regime and the rms velocity is decreased to about $0.6 \mathrm{~m} / \mathrm{s}$. In all cases the inlet velocity is chosen to be equal to the laminar flame speed $s_{L}^{0}$, for each mixture. The typical time based on rms velocity at the flame position and integral length scale is $t_{t}=0.0011 \mathrm{~s}$. In Fig. 20, a typical flame solution of each of the three cases is shown. Small differences exist but the large structures are the same.

In Fig. 21 the flame surface area of the inner reaction layer $A_{f, i l}$ and the integral flame surface area $A_{f, i n t}$ are plotted. The larger the hydrogen content in the fuel, the more the area increases. Also, it shows that there are large differences between the inner layer surface area and the integral surface area, especially in Case-t-A and Case-t-B. This is due to preheat zone deformation, which influences the integral flame surface area a little but only marginally influences the inner layer flame surface 

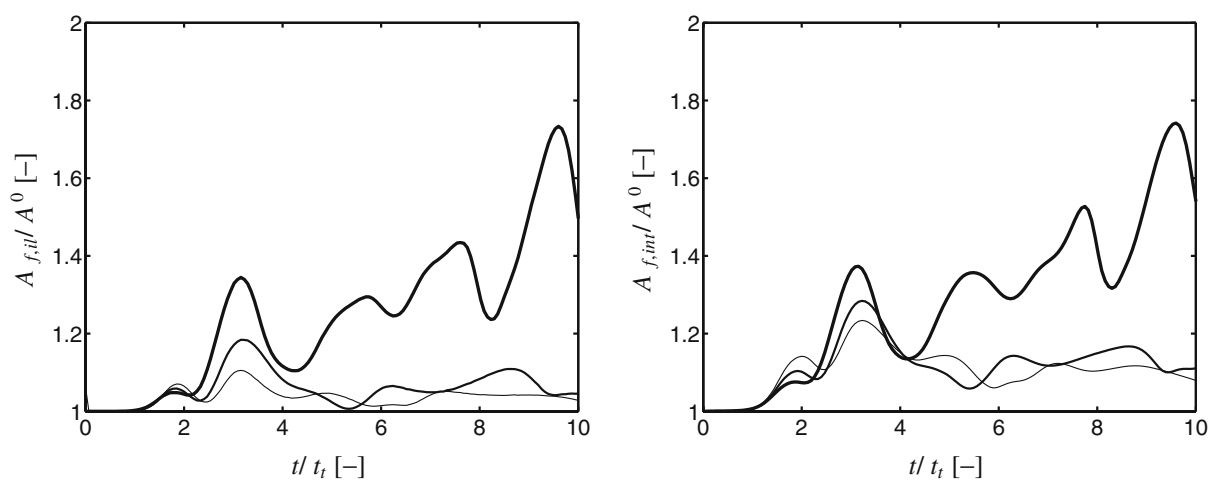

Fig. 21 Flame surface area of inner layer (left) and integral flame surface area (right), with thin line: Case-t-A, medium line: Case-t-B and thick line: Case-t-C as a function of dimensionless time

area. This phenomenon is correlated with the small scales present in the flow, which are able to penetrate the preheat zone, but are damped significantly at the inner layer.

As shown in Fig.22 the integral consumption, $m_{T}$ becomes larger and smaller depending on the flow. Approximately the same peaks are observed as those that appear in the inner layer flame surface area $A_{f, i l}$. The large increase in flame surface area and integral mass consumption in Case-t-C, can be explained by looking at the average flame position. The flame moves fast towards the inlet and encounters stronger turbulence, while Case-t-A and Case-t-B remain at the almost same position and the area increase and the consumption increase do not show large changes in time. When the inner layer flame surface area is plotted against the integral mass
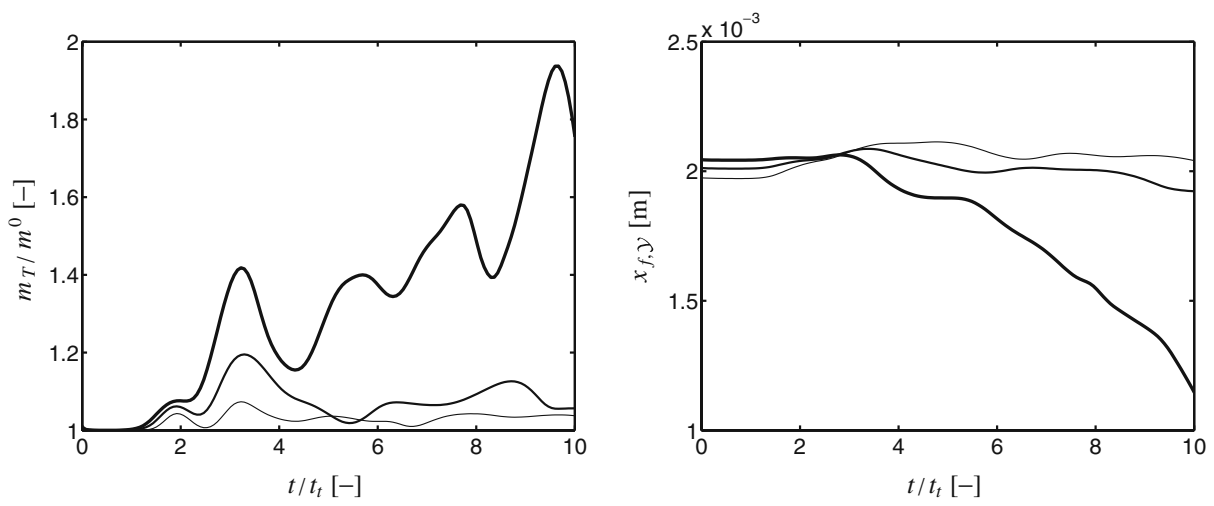

Fig. 22 Integral mass consumption (left) and mean flame position determined based on a volume integration over the progress variable (right), with thin line: Case-t-A, medium line: Case-t-B and thick line: Case-t-C as a function of dimensionless time 

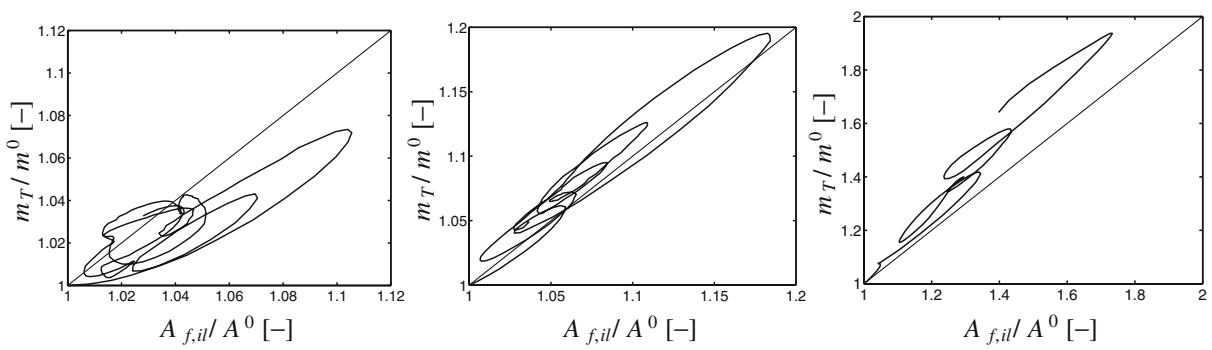

Fig. 23 Turbulent consumption as a function of flame surface area of inner reaction layer (left: Caset-A, middle: Case-t-B and right: Case-t-C)

consumption (Fig. 23), it is observed that there is no linear trend anymore and in all three cases the integral consumption is different from the flame surface area.

Then, individual flamelets are tracked and this yields a local flame stretch and curvature field and a local mass burning rate, which can be plotted as shown in Fig. 24. In comparison with the flame vortex simulations presented in the previous section, more variation is found in these figures. The Markstein numbers are of the same order as in the flame-vortex interactions. However, since in the turbulent flame the stretch rates are more or less 'random', the changes in $Z_{j}$ are more randomly distributed resulting in a "random" scatter in $m$. In other words, the Karlovitz integrals can be the same but different values for $\Delta Z_{j}$ result in different values for $m$. From these figures, linear least squares fits were made to find the Markstein numbers. The Markstein numbers are presented in Table 3. In general the mean Karlovitz integral is approximately zero (although exceptions show for Case$\mathrm{t}-\mathrm{A}$ at time I and for Case-t-C at time III), resulting in a mean value for $m_{i l} / m_{i l}^{0}$ of approximately one. Furthermore Table 3 shows that in general the Markstein number becomes smaller when more hydrogen is present in the fuel, as was also observed in the flame-vortex simulations.

From Table 3 it becomes clear that when the hydrogen content in the fuel mixture increases, a larger preferential diffusion effect is seen. The slopes roughly agree with the slopes found in the flame-vortex simulations. The variation in the mass burning rate as a function of Karlovitz integral is larger than in the flame-vortex simulations. This is a result of the small scales that are present in the turbulent flow and that were absent in the flame-vortex interactions.

\subsection{Results with a single-step Arrhenius rate approach}

A direct comparison between a turbulent flame using single-step chemistry and a 2D-FGM shows the differences in (preferential diffusion) effects that are taken into account in these methods. At the same time also the differences in source term distribution between the different methods is taken into account. Note that for the single-step Arrhenius reaction we take parameters that result in equal burning rate and flame thickness for the plane flame case. Again a three-dimensional turbulent flame is simulated. A finer grid is used in order to be sure of an accurate flame solution $\left(n_{x}=192, n_{y}=n_{z}=96\right)$. The domain size remains the same as in the turbulent flames presented in the previous section. Due to the changed grid 

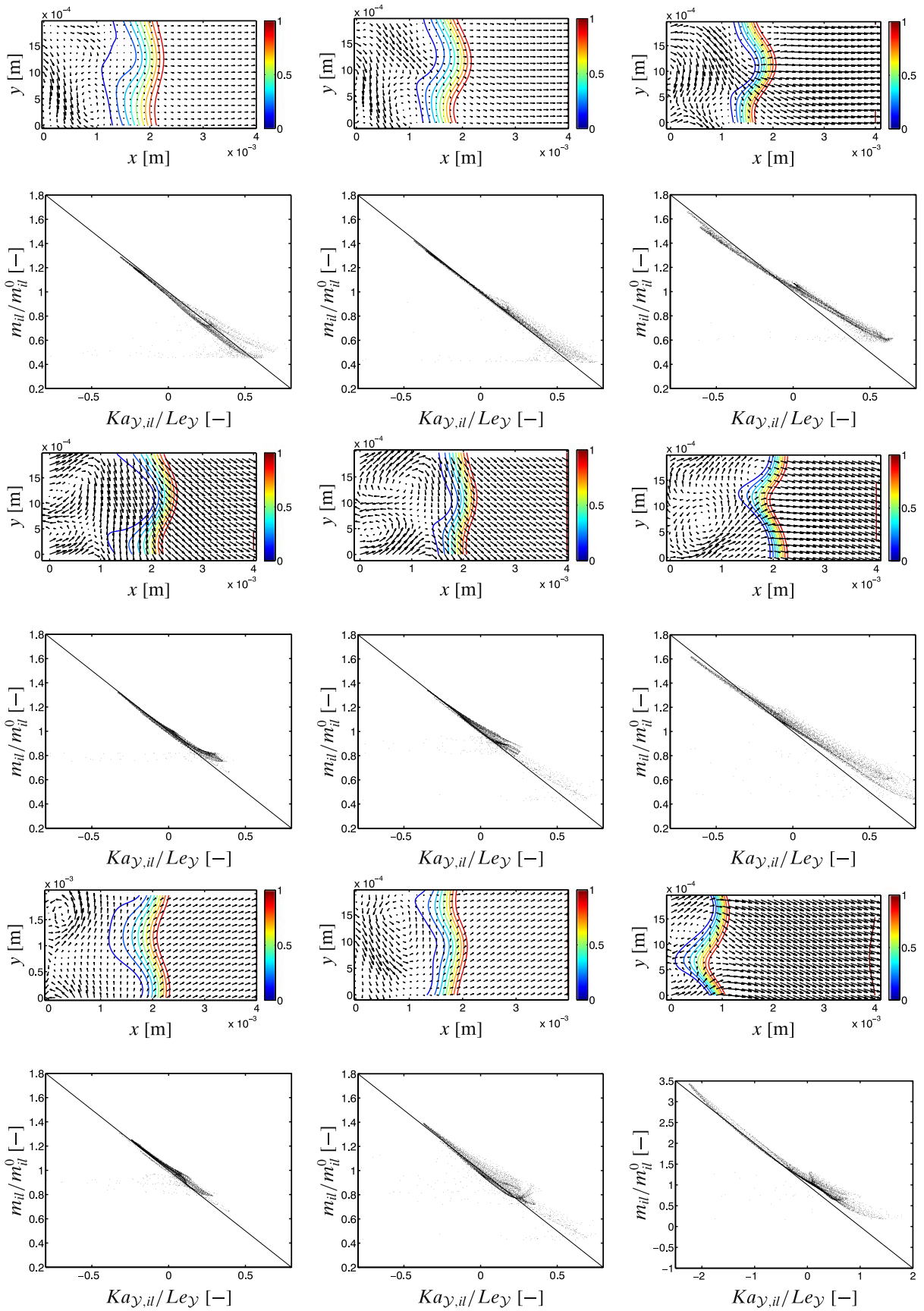

Fig. 24 Flame visualization and flamelet analysis of the different fuel mixtures at $t=3.0 t_{t}$ (top), $t=5.0 t_{t}$ (middle) and $t=10.0 t_{t}$ (bottom) 
Table 3 Mean values of Karlovitz integral, scaled inner layer mass consumption and Markstein number of the three cases at different times

\begin{tabular}{lclc}
\hline & Case-t-A & Case-t-B & Case-t-C \\
\hline $\begin{array}{l}\text { Time I } \\
<K a_{i}>\end{array}$ & 0.14 & 0.11 & \\
$<m_{i} / m^{0}>$ & 0.84 & 0.90 & 0.04 \\
$\mathcal{M}$ & 0.99 & 0.91 & 1.01 \\
Time II & & & 0.76 \\
$\quad<K a_{i}>$ & 0.05 & 0.01 & \\
$<m_{i} / m^{0}>$ & 0.97 & 1.01 & 0.07 \\
$\mathcal{M}$ & 0.82 & 0.86 & 1.00 \\
Time III & & & 0.75 \\
$<K a_{i}>$ & -0.01 & 0.06 & \\
$<m_{i} / m^{0}>$ & 1.03 & 0.96 & 0.13 \\
$\mathcal{M}$ & 0.92 & 0.87 & 0.92 \\
\hline
\end{tabular}

we have slightly different characteristic values for the turbulence, $u_{r m s}=1.29 \mathrm{~m} / \mathrm{s}$ and the longitudinal integral length scale is $L_{f}=7.69 \cdot 10^{-4} \mathrm{~m}$. The longitudinal integral length scale is used as a characteristic turbulence length scale, $l_{t}=L_{f}$. The turbulence time $t_{t}$ becomes $t_{t}=1.54 \cdot 10^{-3} \mathrm{~s}$. Finally, the inflow Kolmogorov scale is equal to $\eta=4.31 \cdot 10^{-5} \mathrm{~m}$.

This field is convected from inlet to flame with $u_{i n}=0.27 \mathrm{~m} / \mathrm{s}$ which is the same as the laminar burning velocity of the used methane/hydrogen mixture $\left(X_{\mathrm{H}_{2}}=0.4\right)$. During this period the turbulent flow decays to $u_{r m s}=0.5 \mathrm{~m} / \mathrm{s}$. The ratio of turbulent and flame velocity is equal to $u_{r m s} / s_{L}^{0} \approx 1.9$. The integral length scale remains approximately constant and combined with a flame thickness of $\delta_{f}=4.7 \cdot 10^{-4} \mathrm{~m}$, this yields $l_{t} / \delta_{f} \approx 1.4$. In the combustion diagram as proposed by e.g. Peters [21], this is situated in the thin reaction zones regime.

Since the largest preferential diffusion effect was seen in the case with $40 \%$ hydrogen in the fuel, this is the case we want to simulate using one-step Arrhenius chemistry. The Lewis number of reactants as well as products is set to $L e_{R}=L e_{P}=$ 0.88 , which follows from definition (15), where the Lewis number of the progress variable is taken at the inner layer position. The models have to be adjusted to match the physics of a flame with a fuel mixture consisting of $60 \%$ methane and $40 \%$ hydrogen. The coefficients that form the $c_{p}$-polynomial are again fitted to data from a simulation with detailed chemistry (and $X_{\mathrm{H}_{2}}=0.4$ ). Also, the coefficients of the $\lambda / c_{p}$-polynomial are adjusted according to,

$$
\lambda / c_{p}=2.71 \cdot 10^{-5}(T / 298 \mathrm{~K})^{0.673} .
$$

By setting the enthalpies of formation of reactants and products, an adiabatic temperature of $T_{a d}=1871 \mathrm{~K}$ is found, which is the same as the value in the detailed case. The chemical model is different, the chemical parameters used in the Arrhenius model are $A=21.81 / \mathrm{s}$ and $E=12990 \mathrm{~J} / \mathrm{mol}$, which results in $m^{0}=0.30 \mathrm{~kg} / \mathrm{m}^{2} \mathrm{~s}$ and the temperature gradient thickness $\delta_{f}=4.7 \cdot 10^{-4} \mathrm{~m}$. With these values the laminar mass burning rate is equal to that of the detailed case. The flame thickness is somewhat smaller than in the detailed case. This is the reason why the grid had to be refined somewhat.

Since preferential diffusion is explicitly shown when plotting the mass burning rate versus the Karlovitz integral, this is the focus of this section. When using singlestep chemistry it is possible to include changes in enthalpy (and thus temperature) 
Fig. 25 Enthalpy of developed flame using 2D-FGM (full line) and using single-step chemistry (dashed line) before the turbulence has an impact on the flame $\left(t=0.3 t_{t}\right)$

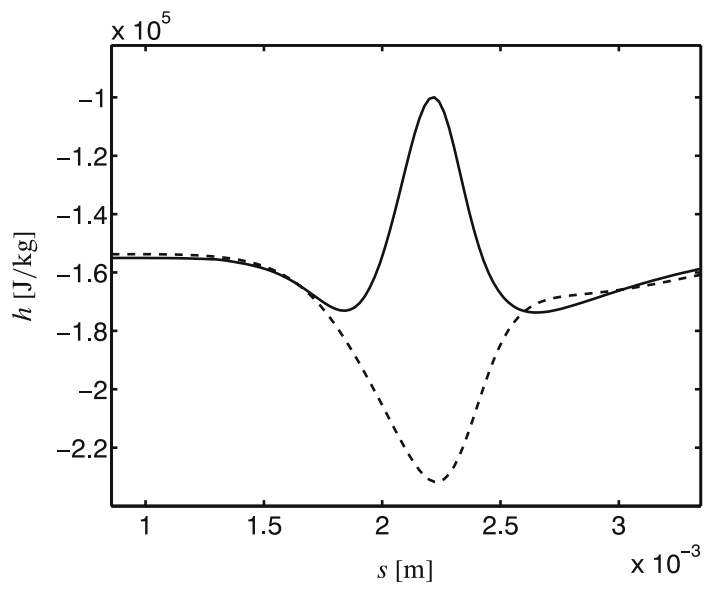

because both $T$ and $Y$ have been solved. However, it is not possible to include changes in local element composition and therefore it is expected that the effects of preferential diffusion are not fully captured. Although the change in local mass burning rate is partly taken into account by a different value of $\rho D$ (determined by $\lambda / c_{p}$ and $L e$ ), it remains uncertain which part of preferential diffusion is captured. Figure 25 shows profiles of enthalpy of developed flames, where the turbulence has not yet reached the flame. This means that flame stretch effects have not affected this solution for the enthalpy.

It can be seen that in the single-step chemistry solution, the enthalpy changes through the flame because of a non-unit Lewis number. However, since only one species is taken into account, a simplified profile is seen. In real flames, the diffusion velocities of all species are different and each species diffusivity can be related to the diffusion rate of energy. In other words, each species has its own Lewis-number, $L e_{i}$. This complex effect is taken into account by the 2D-FGM, where the profile resembles the profile found when using detailed chemistry in a one-dimensional flame.

In Fig. 26, at two different times, the Karlovitz integral is plotted versus the mass burning rate. Almost no deviation from the unity Lewis profile (thin blue line) is seen in the single-step case. The 2D-FGM clearly shows a different slope. The Markstein numbers at the first time are $\mathcal{M}_{S S}=0.99$ and $\mathcal{M}_{F G M}=0.38$ and at the second time

Fig. 26 Inner layer mass burning rate as a function of Karlovitz integral at time $t=2.8 t_{t}$ (left) and $t=4.0 t_{t}$ (right), with black dots: FGM and red dots: single-step chemistry
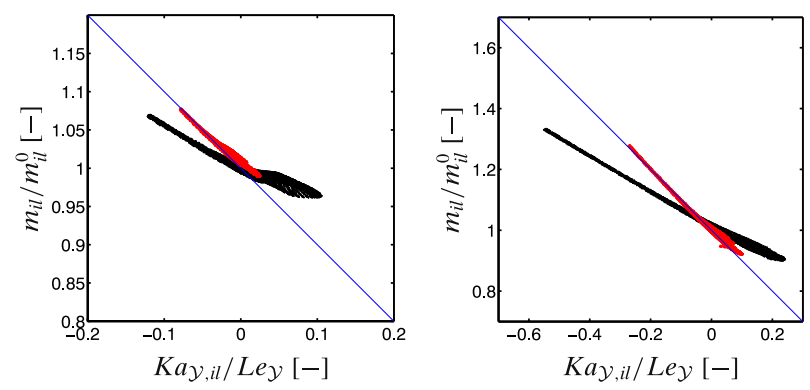
$\mathcal{M}_{S S}=0.95$ and $\mathcal{M}_{F G M}=0.5$. Clearly the slightly larger length scales result in a milder behavior compared to the situation in the previous section; the extent of realizations of Karlovitz integrals is less. The flames are less disturbed giving Markstein values that are much closer to the laminar case as discussed in the beginning of the paper. This also results in the fact that a better resolution shows slightly less scatter. The single-step chemistry simulation is not able to take into account changes in local elemental composition. In [27] it is shown that the element mass fraction of hydrogen $Z_{H}$ and that of carbon $Z_{C}$ are most important. Although the singlestep simulation can predict local changes in enthalpy, it does not give a significant deviation from $\mathcal{M} \approx 1$. This confirms the idea that the changing local conditions caused by preferential diffusion play an important role in the local combustion process. Note that in principle single step chemistry can predict thermo-diffusive instabilities, but in that case for a certain physical fuel, the Lewis number of the progress variable should be defined in a consistent way as worked out in Section 3.2.

\section{Conclusions}

In this paper the effects of preferential diffusion are researched in premixed combustion of mixtures of hydrogen and methane with air. Preferential diffusion in combination with flame stretch and curvature will result in changing burning velocities compared to the unperturbed situation. In turbulent cases the flames are constantly curved and stretched by the flow. The objective is to quantify these effects in terms of global turbulent burning rates in numerical simulation of turbulent premixed combustion. To that end the study is focussed on local laminar burning at physical relevant stretch and curvature conditions. These conditions should be reproduced by the chemical model that is used. Of course this can be calculated by applying detailed chemistry but for most applications this is much to expensive. Here we studied the requirements, implementation and behavior of FGM for the mixtures mentioned. The mixtures were defined by three hydrogen mass fractions of $X_{\mathrm{H}_{2}}=0.0, X_{\mathrm{H}_{2}}=0.2$ and $X_{\mathrm{H}_{2}}=0.4$ in the methane and an equivalence ration of $\phi=0.7$. The Markstein number, being the sensitivity of the mass burning rate to stretch, is an important parameter in this study. The thermo-diffusive instability has a large impact on the total surface area and on the integral consumption of a turbulent flame. Therefore also flame surface area and integral consumption are important quantities. In the present study the conditions of the perturbations which are formed by coherent vortices and turbulence are kept mild in order to make careful analyses possible.

First one-dimensional laminar flamelets under the influence of stretch were considered. These flamelets were calculated with detailed chemistry. It was found that the burning rate can be altered quite strongly due to preferential diffusion at relevant stretch rates. Especially for the highest amount of hydrogen the difference with stretched methane flames (which have almost no preferential diffusion) becomes much more pronounced compared to the $20 \%$ case. For both mixtures, the sensitivity of the mass burning rate to stretch diminishes.

After setting these reference results we proposed a way to include preferential diffusion effects in FGM. A general framework is set-up and it was argued that at least two control variables are needed to include the mentioned effects up to first 
order. These variables consisted of a progress variable as a main flame coordinate and a second controlling variable to describe perturbations. These were defined such that a large sensitivity is reached with the manifold lookup. Furthermore the database was constructed in which, besides source terms of the controlling variables, also diffusion coefficients are tabulated. Besides this Lewis numbers of controlling variables were defined explicitly.

With this method, implemented in the numerical DNS-code SENGA, twodimensional simulations were carried out in which a flame front was subjected to coherent vortices. It was found that Markstein numbers different from 1 could be reproduced, decreasing with increasing hydrogen content. This in contrast to computations with a 1D manifold and one controlling variable in which Markstein numbers were obtained that did not significantly deviate from unity. It was also found that with the 2D manifold the flame surface area did not periodically return to the minimum value as it does when no preferential diffusion is taken into account; this might be a manifestation of thermo-diffusive instability. Therefore we conclude that we improved the FGM model for including preferential diffusion effects. Moreover an analysis was performed to investigate the effects of increasing the dimension of the manifold at stretch and curvature fields found in the the 2D simulations. Here it was also shown that use of a 2D manifold gives significantly improved predictions compared to the use of a 1D manifold and it approaches results with detailed chemistry. Another analysis showed that remaining errors are of the same order as errors that are caused by the flamelet assumption and numerical implementation. Additionally it was found that the enthalpy changes with stretch are not ideally represented.

In order to introduce a broader spectrum of disturbances and more physical turbulent behavior also three-dimensional computations were carried out. Although smaller flow structures were introduced, the range in local stretch rates was comparable to the stretches found in the flame-vortex interactions and the Markstein numbers that were typically found did not change significantly from those found in two-dimensional simulations. Moreover for a turbulent case with slightly larger flow structures it was found that simulations with Arrhenius one-step chemistry with $40 \%$ hydrogen and using the appropriate Lewis number did not result in burning rates associated to physical preferential diffusion effects whereas the same case with the 2D manifold showed a much better behavior.

Acknowledgements This research was funded by Stichting Technische Wetenschappen, STW (Dutch Science Foundation), grant number EWO.5874 and supported by Nederlandse Supercomputer Faciliteiten, NCF (Dutch Supercomputer Facilities).

Open Access This article is distributed under the terms of the Creative Commons Attribution Noncommercial License which permits any noncommercial use, distribution, and reproduction in any medium, provided the original author(s) and source are credited.

\section{Appendix: Application of a 1D Manifold}

Two-dimensional flame-vortex interactions

In this appendix, two-dimensional vortex-flame interactions are studied using a one-dimensional manifold. The flow conditions are as given in the reference case, 

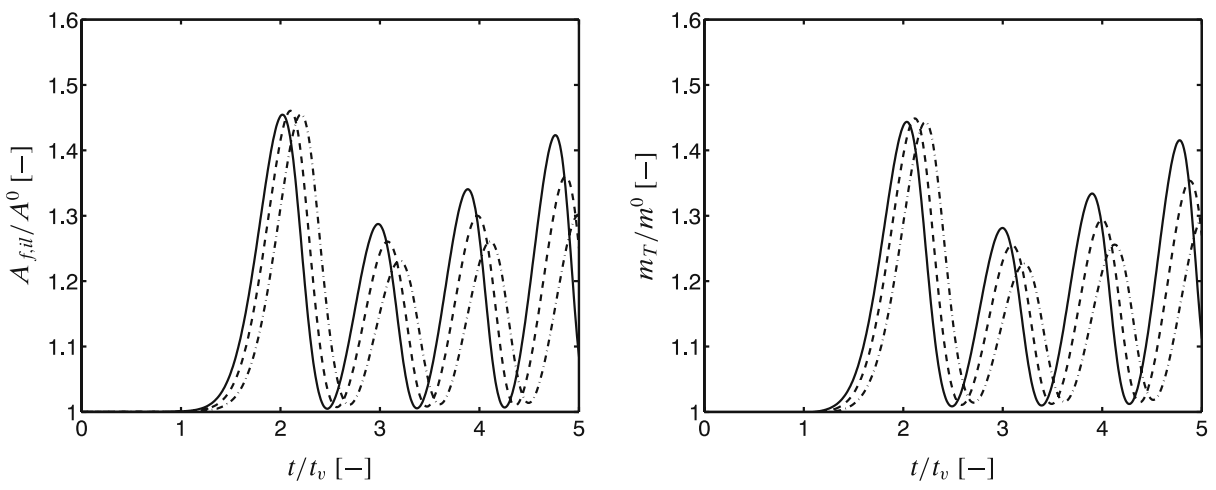

Fig. 27 Flame surface area of reaction layer (left) and mass consumption (right) for a 1D-FGM simulation with full line: Val-v-A, dashed line: Val-v-B and dash-dotted line: Val-v-C

described in Section $4.1\left(A_{w}=0.6 \mathrm{~m} / \mathrm{s}\right.$ and $\left.k_{0}=2501 / \mathrm{m}\right)$. In this section, onedimensional manifolds with conserved enthalpy and elemental composition are used. Thus the influence of physical occuring local changes in elemental composition and enthalpy on the source term are not taken into account. Only information from a single one-dimensional flame solution is stored and no deviations from this single flamelet are included. Here it will be investigated whether preferential diffusion effects appear in these simulations. Simulations with the three different fuel mixtures will be indicated by Val-v-A $\left(X_{\mathrm{H}_{2}}=0.0\right)$, Val-v-B $\left(X_{\mathrm{H}_{2}}=0.2\right)$ and Val-v-C $\left(X_{\mathrm{H}_{2}}=0.4\right)$.

First, global features of the flame, such as flame surface area, integral mass burning rate and the relation between these two variables is investigated and results are shown in Figs. 27, 28, 29 and 30. Flame surface area and mass burning rate are key features. The vortex time $t_{v}$ is defined as given in Eq. 27. Then, local features are
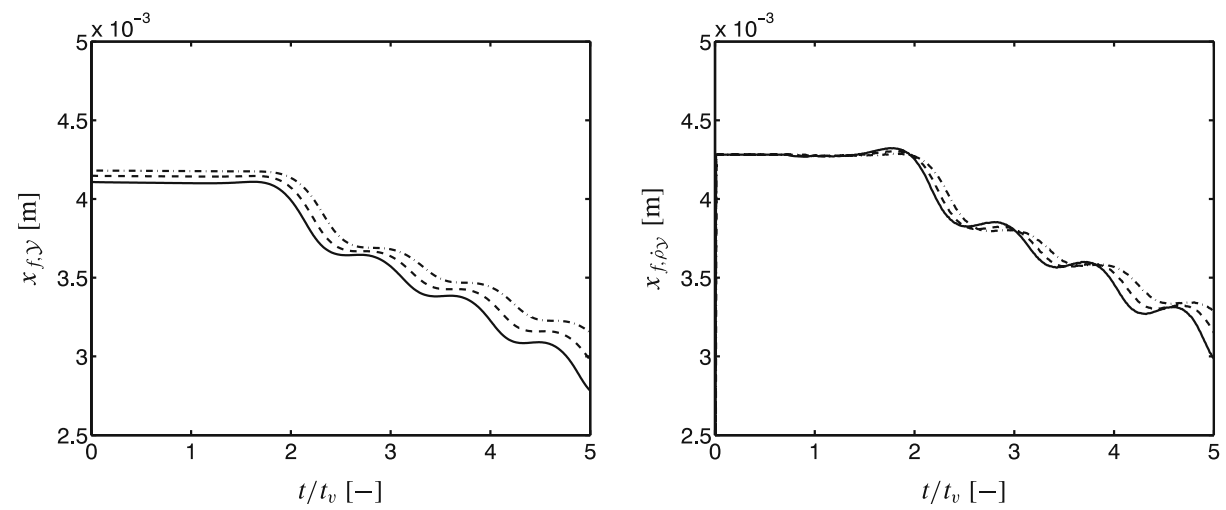

Fig. 28 Flame position based on reaction progress variable (left) and based on position of the peak of the chemical source term (right) for a 1D-FGM simulation with full line: Val-v-A, dashed line: Val-v-B and dash-dotted line: Val-v-C 

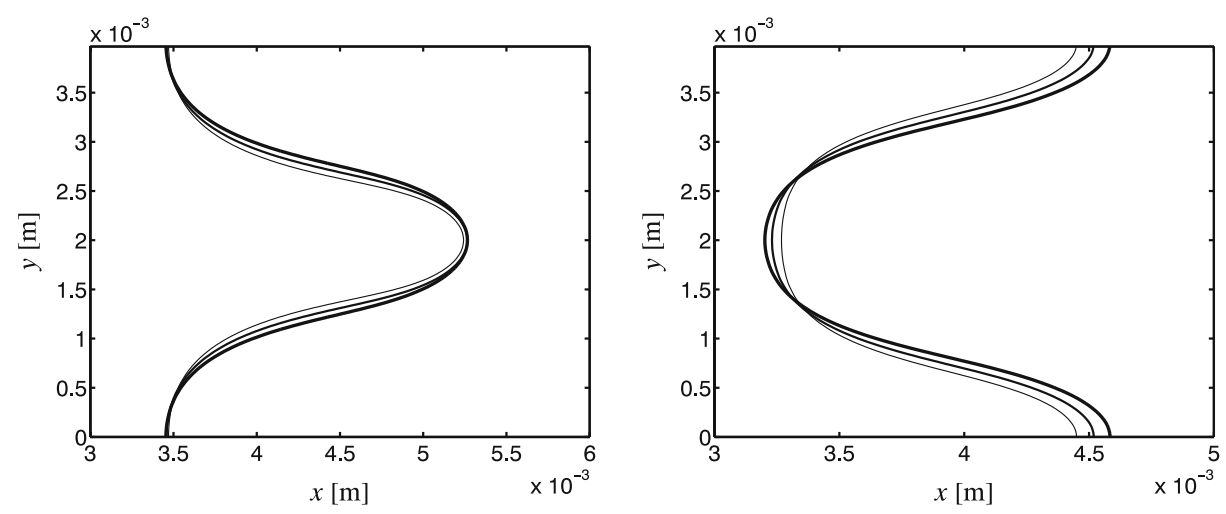

Fig. 29 Iso-contour of inner layer progress variable at the first peak of each case (left figure) and at the second peak of each case (right figure), with thickest line Val-v-A, medium line Val-v-B and thinnest line Val-v-C

investigated. The relation between the dimensionless flame stretch rate, represented by the Karlovitz integral, and local mass burning rates, as explained in Section 4.2, are shown in Figs. 31 and 32. From this relation, the Markstein number can be derived.

In Fig. 27 the inner layer flame surface area and the integral mass consumption are displayed. It can be seen that differences exist in peak height as well as peak position. The differences in peak height are correlated with the mean flame position, as is shown in Fig. 28. Val-v-A moves faster towards the inlet and therefore encounters stronger vortices and this results in a larger displacement from the initial (flat) flame position. The mean inflow velocity is set to exactly balance the propagation speed for flat flames for each of the fuel mixtures. Also, the flames are initially positioned with the maximum of their chemical source term at the same position. Since the flame structure is different (as shown in Fig. 3, right), the chemical source distribution is different and this causes differences in the reaction of the flame to the vortical flow. In order to show the geometrical differences in flame solution, in Fig. 29 the inner layer iso-contour of the three flame solutions are shown at times corresponding to the
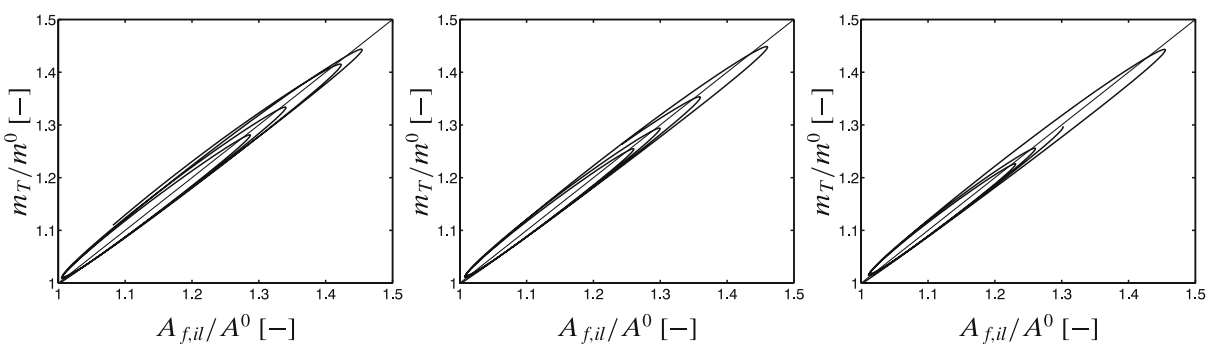

Fig. 30 Mass consumption versus flame surface area of reaction layer with left: Val-v-A, middle: Val-v-B and right: Val-v-C 

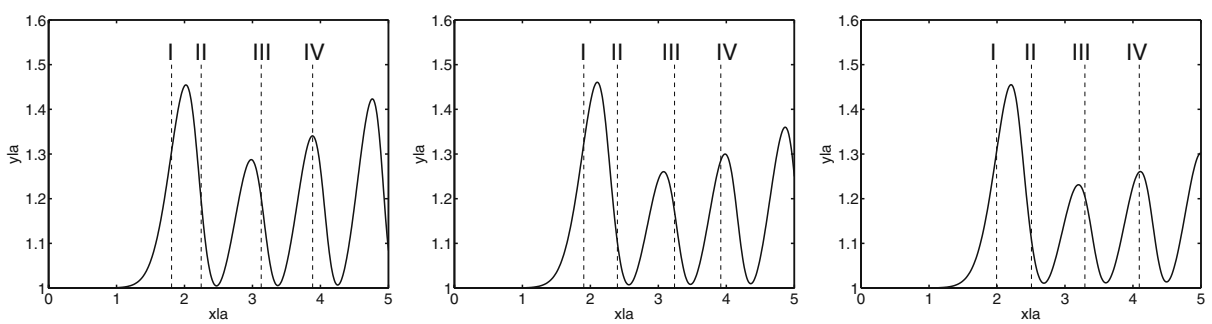

Fig. 31 Scaled flame surface area as a function of time of case Val-v-A (left), case Val-v-B (middle) and case Val-v-C (right), where four different times are indicated. On these times, the Karlovitz integral and inner layer mass consumption will be investigated

first peak and the second peak in Fig. 27. Especially in the right figure, corresponding to the second peak in Fig. 27, it is found that the inner layer iso-contour of Val-v-A is narrower than the other profiles and this is associated with a larger increase in flame surface area and integral mass consumption.

Now, it is investigated whether a linear relation exists between the flame surface area and the integral consumption. Preferential diffusion effects are not taken into account (i.e. there is only one controlling variable) and for a flame without preferential diffusion (and infinitely thin reaction layer) a linear relation is expected. This is shown in Fig. 30 and it can be observed that there is very little difference between the cases. The integral consumption scales approximately linear with flame surface area for all cases and the data do collapse almost exactly to a single straight line.

In the presented figures it can be observed that for the different fuels no intrinsically different results are obtained. The behaviour of the cases with hydrogen in the fuel is similar to that with no hydrogen, e.i. only methane. Only the source term is different resulting in different propagation speeds. All cases return periodically to flat flame conditions. Such a behaviour would not be possible when thermo-diffusive effects play a role. It can be concluded that with the use of a one-dimensional manifold no preferential diffusion effects are observed and a $L e=1$ behaviour is observed.

Finally, at different times in the simulation (as displayed in Fig. 31, the flame solutions will be compared by performing flamelet analysis, and plotting the Karlovitz integral versus the inner layer mass consumption, as explained in Section 4.2. Since the velocity-fields prescribed at the inlet are identical, the differences in flame solutions have to come from different chemical effects. The flame stretch rates are a result of a two-way coupling between flow and chemistry. When the preferential diffusion effect is active, a different slope is expected for the different fuel mixtures as becomes clear from (1) and (2). Adding hydrogen to the fuel influences the Markstein number. However, because one-dimensional flamelet databases are used, the effects of flame stretch on local composition is not taken into account. Indeed, Fig. 32 shows that a slope of approximately minus one is found in all fuel mixtures. This confirms the expectation that when a one-dimensional manifold is used, it is not 

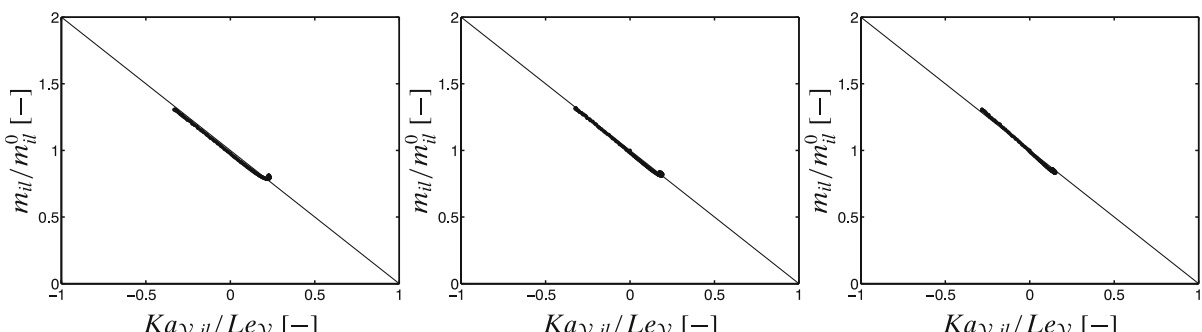

$K a_{y, i l} / L e_{y}[-]$
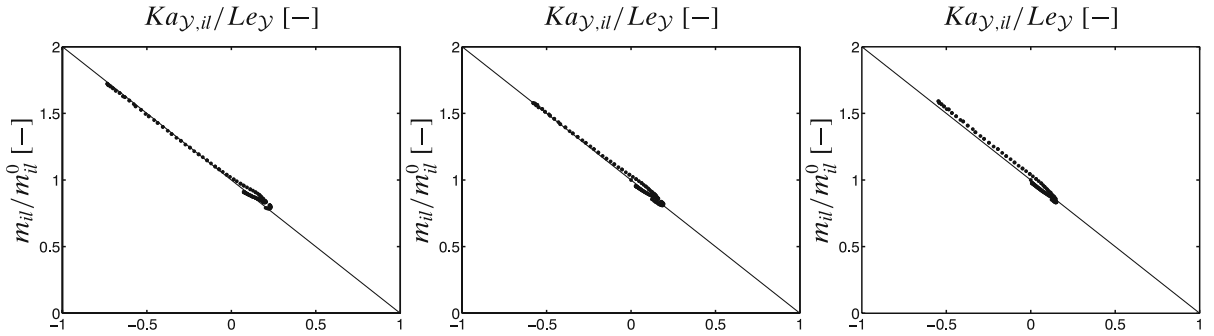

$K a_{\mathcal{Y}, \text { il }} / L e_{\mathcal{Y}}[-]$

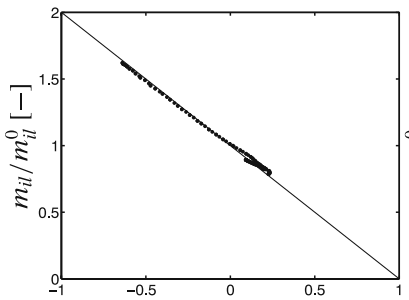

$K a_{\mathcal{Y}, i l} / L e_{\mathcal{Y}}[-]$

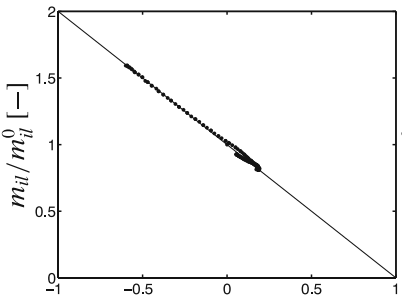

$K a_{\mathcal{Y}, i l} / L e_{\mathcal{Y}}[-]$

$K a_{\mathcal{Y}, i l} / L e_{\mathcal{Y}}[-]$

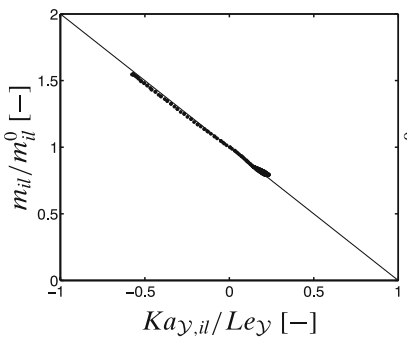

$K a_{\mathcal{Y}, i l} / L e_{\mathcal{Y}}[-]$

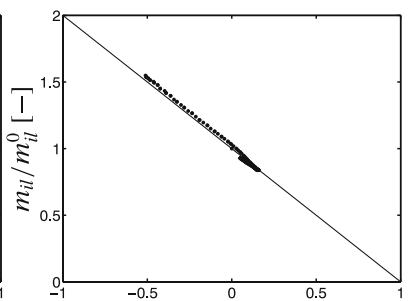

$K a_{\mathcal{Y}, i l} / L e_{\mathcal{Y}}[-]$
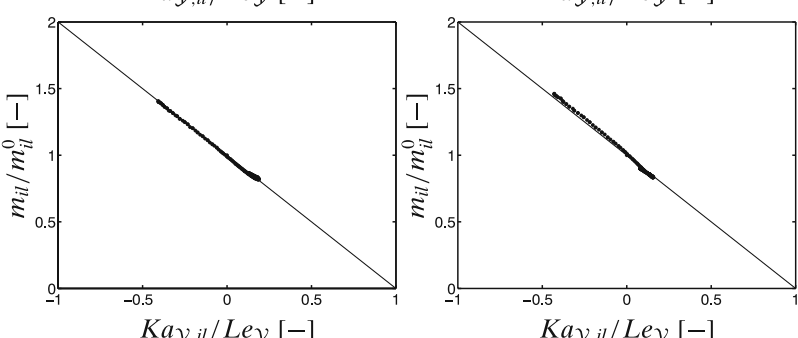

Fig. 32 Scaled inner layer consumption as a function of Karlovitz integral for case Val-v-A (left), case Val-v-B (middle) and case Val-v-C (right) at times (top to bottom) I to IV

possible to capture the preferential diffusion effect in stretched flames. In Table 4 the mean value of the Karlovitz integral and the mean value of the scaled inner layer mass burning rate are shown. The mean Karlovitz integral is approximately zero in all cases and the mean scaled inner layer mass consumption is approximately one. This indicates that the effects of positive and negative stretch balance, as expected.

Flamelet analysis showed that when a $1 \mathrm{D}-\mathrm{FGM}$ is used, the slopes $(\mathcal{M})$ vary slightly between the three cases. Since the flamelet database is only one-dimensional, 
Table 4 Mean values of Karlovitz integral, scaled inner layer mass consumption and Karlovitz numbers of the three cases Val-v-A, Val-v-B and Val-v-C at different times

\begin{tabular}{|c|c|c|c|}
\hline & Val-v-A & Val-v-B & Val-v-C \\
\hline \multicolumn{4}{|l|}{ Time I } \\
\hline$<K a_{\mathcal{Y}, i l} / L e_{\mathcal{Y}}>$ & 0.05 & 0.04 & 0.03 \\
\hline$<m_{i l} / m_{i l}^{0}>$ & 0.95 & 0.96 & 0.96 \\
\hline $\mathcal{M}$ & 0.94 & 0.99 & 1.12 \\
\hline \multicolumn{4}{|l|}{ Time II } \\
\hline$<K a_{\mathcal{Y}, i l} / L e_{\mathcal{Y}}>$ & 0.02 & 0.00 & 0.00 \\
\hline$<m_{i l} / m_{i l}^{0}>$ & 1.03 & 1.01 & 1.01 \\
\hline $\mathcal{M}$ & 0.96 & 1.00 & 1.09 \\
\hline \multicolumn{4}{|l|}{ Time III } \\
\hline$<K a_{\mathcal{Y}, i l} / L e_{\mathcal{Y}}>$ & 0.00 & 0.01 & 0.01 \\
\hline$<m_{i l} / m_{i l}^{0}>$ & 1.01 & 1.00 & 0.99 \\
\hline $\mathcal{M}$ & 0.94 & 0.98 & 1.09 \\
\hline \multicolumn{4}{|l|}{ Time IV } \\
\hline$<K a_{\mathcal{Y}, i l} / L e_{\mathcal{Y}}>$ & 0.01 & 0.03 & 0.01 \\
\hline$<m_{i l} / m_{i l}^{0}>$ & 0.99 & 0.97 & 0.98 \\
\hline $\mathcal{M}$ & 0.95 & 0.99 & 1.10 \\
\hline
\end{tabular}

the local element composition and enthalpy are not taken into account. However, from simulations using detailed chemistry, e.g. [20] and as seen in Section 2, a larger preferential diffusion effect is expected. Therefore, in the main results section, flamelet databases are used including changes in $Z_{j}$ and $h$, to investigate whether a two-dimensional manifold is able to capture the full preferential diffusion effect in stretched flames.

\section{References}

1. Chakraborty, N., Cant, R.S.: Influence of lewis number on curvature effects in turbulent premixed flame propagation in the thin reaction zones regime. Phys. Fluids 17, 105105 (2005)

2. Chakraborty, N., Cant, R.S.: Influence of lewis number on strain rate effects in turbulent premixed flame propagation. Int. J. Heat Mass Transfer 49, 2158-2172 (2006)

3. Chakraborty, N., Cant, R.S.: Effects of Lewis number on turbulent scalar transport and its modelling in turbulent premixed flames. Combust. Flame 156(7), 1427-1444 (2009)

4. CHEM1D: A One-Dimensional Laminar Flame Code. Eindhoven University of Technology. www.combustion.tue.nl/chem1d. Accessed 19 July 2010

5. Dunstan, T.D., Jenkins, K.W.: The effects of hydrogen substitution on turbulent premixed methane-air kernels using direct numerical simulation. Int. J. Hydrog. Energy 34, 8389-8404 (2009)

6. Eggels, R.L.G.M., de Goey, L.P.H.: Mathematically reduced reaction mechanisms applied to adiabatic flat hydrogen/air flames. Combust. Flame 100, 559-570 (1995)

7. Gauducheau, J.L., Denet, B., Searby, G.: A numerical study of lean CH4/H2/air premixed flames at high pressure. Combust. Sci. Technol. 137, 81-99 (1998)

8. Gicquel, O., Darabiha, N., Thévenin, D.: Laminar premixed hydrogen/air counterflow flame simulations using flame prolongation of ILDM with differential diffusion. Proc. Combust. Inst. 28, 1901-1908 (2000)

9. de Goey, L.P.H., ten Thije Boonkkamp, J.H.M.: A mass-based definition of flame stretch for flames of finite thickness. Combust. Sci. Technol. 122, 399-405 (1997)

10. de Goey, L.P.H., ten Thije Boonkkamp, J.H.M.: A flamelet description of premixed laminar flames and the relation with flame stretch. Combust. Flame 119, 253-271 (1999)

11. Halter, F., Chauveau, C., Gökalp, I.: Characterization of the effects of hydrogen addition in premixed methane/air flames. Int. J. Hydrog. Energy 32, 2585-2592 (2007)

12. Hawkes, E.R., Chen, J.H.: Direct numerical simulation of hydrogen-enriched lean premixed methane-air flames. Combust. Flame 138, 242-258 (2004) 
13. Haworth, D.C., Poinsot, T.J.: Numerical simulations of lewis number effects in turbulent flames. J. Fluid Mech. 244, 405-436 (1992)

14. Hu, E., Huang, Z., He, J., Jin, C., Zheng, J.: Experimental and numerical study on laminar burning characteristics of premixed methane-hydrogen-air flames. Int. J. Hydrog. Energy 34, 4876-4888 (2009)

15. Jenkins, K.W., Cant, R.S.: DNS of turbulent flame kernels. In: Proceedings Second AFOSR Conference on DNS and LES, pp. 192-202. Kluwer Academic Publishers (1999)

16. Maas, U., Pope, S.B.: Simplifying chemical kinetics: intrinsic low-dimensional manifolds in composition space. Combust. Flame 88, 239-264 (1992)

17. van Oijen, J.A.: Flamelet-generated manifolds: development and application to premixed laminar flames. Ph.D. thesis, Technische Universiteit Eindhoven, Eindhoven, The Netherlands (2002)

18. van Oijen, J.A., Bastiaans, R.J.M., Groot, G.R.A., de Goey, L.P.H.: Direct numerical simulations of premixed turbulent flames with reduced chemistry: Validation and flamelet analysis. Flow Turbul. Combust. 75, 67-84 (2005)

19. van Oijen, J.A., de Goey, L.P.H.: Modelling of premixed laminar flames using flamelet-generated manifolds. Combust. Sci. Technol. 161, 113-138 (2000)

20. van Oijen, J.A., Ramaekers, W.J.S., de Goey, L.P.H.: Predicting pollutant formation with steady flamelet models. In: Proceedings of the12th International Conference on Numerical Combustion, Monterey, CA, United States (2008)

21. Peters, N.: Turbulent Combustion. Cambridge University Press, Cambridge (2000)

22. Rutland, C.J., Trouvé, A.: Direct simulations of premixed turbulent flames with nonunity lewis numbers. Combust. Flame 94, 41-57 (1993)

23. Sankaran, R., Hawkes, E.R., Chen, J.H., Lu, T., Law, C.K.: Structure of a spatially developing turbulent lean methane-air bunsen flame. Proc. Combust. Inst. 31, 1291-1298 (2007)

24. Smith, G.P., Golden, D.M., Frenklach, M., Moriarty, N.W., Eiteneer, B., Goldenberg, M., Bowman, C.T., Hanson, R.K., Song, S. Gardiner, W.C. Jr., Lissianski, V.V., Qin, Z.: www.me. berkeley.edu/gri-mech/. Accessed 19 July 2010

25. Somers, L.M.T.: The simulation of flat flames with detailed and reduced chemical models. Ph.D. thesis, Technische Universiteit Eindhoven, Eindhoven, The Netherlands (1994)

26. de Swart, J.A.M., Groot, G.R.A., van Oijen, J.A., ten Thije Boonkkamp, J.H.M., de Goey, L.P.H.: Detailed analysis of the mass burning rate of stretched flames including preferential diffusion effects. Combust. Flame 145, 245-258 (2006)

27. de Swart, J.A.M.: Modeling and analysis of flame stretch and preferential diffusion in premixed flames. Ph.D. thesis, Technische Universiteit Eindhoven, Eindhoven, The Netherlands (2009)

28. Trouve, A., Poinsot, T.J.: The evolution equation for the flame surface density. J. Fluid Mech. 278, 1-31 (1994)

29. Vreman, A.W., Albrecht, B.A., van Oijen, J.A., de Goey, L.P.H., Bastiaans, R.J.M.: Premixed and non-premixed generated manifolds in large-eddy simulation of sandia flame D and F. Combust. Flame 153(3), 394-416 (2008)

30. Vreman, A.W., van Oijen, J.A., de Goey, L.P.H., Bastiaans, R.J.M.: Direct numerical simulation of hydrogen addition in turbulent premixed bunsen flames using flamelet generated manifold reduction. Int. J. Hydrog. Energy 34, 2778-2788 (2009)

31. Warnatz, J., Maas, U., Dibble, R.W.: Combustion. Springer, Berlin (1996)

32. Yu, G., Law, C.K., Wu, C.K.: Laminar flame speeds of hydrocarbon + air mixtures with hydrogen addition. Combust. Flame 63, 339-347 (1986) 